\title{
Spatial Coevolution for Generative Adversarial Network Training
}

\author{
ERIK HEMBERG, JAMAL TOUTOUH, ABDULLAH AL-DUJAILI, \\ TOM SCHMIEDLECHNER, and UNA-MAY O'REILLY, MIT CSAIL, USA
}

Generative Adversarial Networks (GANs) are difficult to train because of pathologies such as mode and discriminator collapse. Similar pathologies have been studied and addressed in competitive evolutionary computation by increased diversity. We study a system, Lipizzaner, that combines spatial coevolution with gradient-based learning to improve the robustness and scalability of GAN training. We study different features of Lipizzaner's evolutionary computation methodology. Our ablation experiments determine that communication, selection, parameter optimization, and ensemble optimization each, as well as in combination, play critical roles. Lipizzaner succumbs less frequently to critical collapses and, as a side benefit, demonstrates improved performance. In addition, we show a GAN-training feature of Lipizzaner: the ability to train simultaneously with different loss functions in the gradient descent parameter learning framework of each GAN at each cell. We use an image generation problem to show that different loss function combinations result in models with better accuracy and more diversity in comparison to other existing evolutionary GAN models. Finally, Lipizzaner with multiple loss function options promotes the best model diversity while requiring a large grid size for adequate accuracy.

CCS Concepts: • Computing methodologies $\rightarrow$ Neural networks; Bio-inspired approaches; Unsupervised learning;

Additional Key Words and Phrases: Generative adversarial networks, coevolution, diversity

ACM Reference format:

Erik Hemberg, Jamal Toutouh, Abdullah Al-Dujaili, Tom Schmiedlechner, and Una-May O’Reilly. 2021. Spatial Coevolution for Generative Adversarial Network Training. ACM Trans. Evol. Learn. Optim. 1, 2, Article 6 (July 2021), 28 pages.

https://doi.org/10.1145/3458845

\section{INTRODUCTION}

Generative modeling aims to learn a function that describes a latent, i.e., unknown, distribution. In a common setup, generative models undergo gradient-based training using observations from the latent distribution and they learn either an estimate of this distribution (explicit density) or how

This research was partially funded by European Union's Horizon 2020 research and innovation program under the Marie Skłodowska-Curie grant agreement No 799078, by the MINECO and FEDER projects TIN2016-81766-REDT and TIN201788213-R and the Systems that Learn Initiative at MIT CSAIL.

Authors' addresses: E. Hemberg, J. Toutouh, A. Al-Dujaili, T. Schmiedlechner, and U.-M. O.Reilly, MIT Computer Science \& Artificial Intelligence Laboratory, 32 Vassar Street, Cambridge, MA 02139, USA; emails: hembergerik@csail.mit.edu, toutouh@mit.edu, ash.aldujaili@gmail.com, tschmied@mit.edu, unamay@csail.mit.edu.

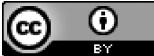

This work is licensed under a Creative Commons Attribution International 4.0 License.

(c) 2021 Association for Computing Machinery.

2688-299X/2021/07-ART6 \$15.00

https://doi.org/10.1145/3458845

ACM Transactions on Evolutionary Learning and Optimization, Vol. 1, No. 2, Article 6. Pub. date: July 2021. 
to generate samples from it (implicit density). The trained model can then be used to generate unobserved samples.

Generative Adversarial Networks (GANs) consist of two neural network models: a discriminator and a generator [Goodfellow 2016]. GANs use an adversarial paradigm. The discriminator's training objective is to distinguish real samples in the training data set from samples synthesized by the generator. The training objective of the generator is to fool the discriminator with its samples, synthesized from a latent input space and a non-linear function. This forms a coupled minimization and maximization "minimax" problem. The adversarial paradigm introduces GAN training challenges. During unsuccessful training, the networks' parameters stabilize into a sub-optimal equilibrium or continually oscillate between sub-optimal performance levels. After successful training, the generator serves as the generative model [Goodfellow 2016].

In this contribution, we study Lipizzaner, a system originally designed and demonstrated to reliably address the degenerate behaviors of mode collapse (the generator output converges to a single mode, e.g., the digit 1, when trying to learn how to generate images of the digits 0 to 9) and discriminator collapse (the discriminator converges to a single mode, e.g., only discriminating the image of digit 1) [Al-Dujaili et al. 2018a; Schmiedlechner et al. 2018; Toutouh et al. 2019] and efficiently yield generative models; for related work, see Section 2. Lipizzaner combines spatially distributed coevolution and GAN training; see Section 3 for notation and Section 4 for the method. Lipizzaner consists of an asynchronous competitive coevolutionary algorithm executing on a 2D spatial grid of cells organized into overlapping neighborhoods. Two competitive coevolving sub-populations exist on each cell-one of generators and the other of discriminators, collected from the cell and its adjacent neighbors. Training occurs pairwise across the sub-populations. The neural network models are updated with stochastic gradient descent (SGD) using the minimax objective, following conventional machine learning methods. The training parameters such as learning rate of SGD are learned with an evolution-based method. Between training epochs, the competing sub-populations are updated with copies of best neural network models from the cell's local neighborhood. This is a form of implicit communication that relies upon the overlap of the cell neighborhoods. The solutions at each individual cell improve because of the training, while the epoch-by-epoch spatial propagation of best solutions prevents local pathologies from persisting. Combined, this approach ensures robustness.

While Lipizzaner [Al-Dujaili et al. 2018a] has many typical features of evolutionary computation, it also has many features specific to distributed coevolution on a spatial grid. These features include spatial communication, local (sub-population) selection, plus the evolution of generator ensemble weights and the initial value of its networks' learning rate under SGD, which is central to GANs. We explore Lipizzaner empirically in Section 5. Our first question, RQ-1, answered through ablation, is whether each of these different features of Lipizzaner's evolutionary computation methodology are needed and helpful (see Section 5.2).

Generally, the ideal loss functions for GAN training are unknown. In seeking the best model that spans the entire latent distribution, a developer typically has to train with each of a suite of possible loss functions multiple times due to sensitivity to initial conditions. Parameter training arguably could benefit from different loss functions at different times during training, but these are unknown. Lipizzaner has the ability to train simultaneously with different loss functions for each GAN at each cell. We therefore ask a second question (see Section 5.3, RQ-2): Do different loss function combinations result in generative models with better performance and higher diversity of solutions and model parameters? We draw upon four different loss functions: See Section 3 to configure six experimental setups of loss function combinations, see Table 5, and compare them. Effectively, using different loss functions explicitly imposes pressure for population-wide diversity in adaptation. We verify this diversity by comparing the parameters of different trained models 
of Lipizzaner. Moreover, we verify whether the diversity in loss function choice results in better coverage of the latent space. We deliberately choose an image generation problem that allows us to gauge total variational distance [Li et al. 2017a] and the coverage of every class in the image set. In addition, we measure the generative model performance with Frechet Inception Distance (FID) score [Heusel et al. 2017a].

We extend previous work on Lipizzaner [Al-Dujaili et al. 2018a; Schmiedlechner et al. 2018; Toutouh et al. 2019] and provide the following contributions (see Section 6):

(1) We describe Lipizzaner, a scalable, distributed, GAN training system.

(2) We establish the necessity of spatial communication, local selection, and GAN training parameter evolution toward better performance by Lipizzaner through ablation studies.

(3) We experimentally confirm that combinations of different loss functions, including randomization of loss function choice, while slightly more computationally expensive, are advantageous in the common circumstance of not knowing the ideal loss function. In addition, the trained model parameter and output diversity increases.

\section{RELATED WORK}

We now discuss work related to competitive coevolutionary algorithms for minimax problems (Section 2.1), how evolutionary computation and GANs have been combined in prior work (Section 2.2), scaling evolutionary computation for machine learning (Section 2.3), and common approaches to GAN training (Section 2.4).

\subsection{Coevolutionary Algorithms for Minimax Problems}

There is extended work on coevolutionary algorithms covering multi-objective optimization [Goh and Tan 2009; Miguel Antonio and Coello Coello 2018], active learning [Le Ly and Lipson 2014], large-scale optimization [Zhang et al. 2019a], and strategic decision support [Johnson et al. 2005]. Competitive coevolutionary algorithms have adversarial populations (usually two) that simultaneously evolve solutions against each other [Floreano and Mattiussi 2008; Popovici et al. 2012] with members engaging in two-player games. They employ fitness functions that rate a solution relative to its adversaries and can sometimes be described as a zero-sum game or, more generally, a minimax optimization [Al-Dujaili et al. 2018b; Barbosa 1999, 1997; Herrmann 1999]. In seminal work, Hillis [1990] showed that more efficient sorting programs can be produced by competitively co-evolving them versus their testing programs.

Reasons offered for a limitation are usually accompanied by a general remedy and one or more methods to implement the remedy. For example, one limitation is due to the use of symmetric fitness functions. A remedy for this is asymmetry, i.e., using different fitness functions [Branke and Rosenbusch 2008; Jensen 2003; Qiu et al. 2017]. The equivalent in GAN training is the use of Binary Cross Entropy as a loss metric for a generator and Mean Squared Error as a loss metric for a discriminator. As another example, Cliff and Miller [1995] note intransitive (cyclic) superiority and address it with more specific measures of fitness and genotypes to track the evolutionary dynamics. Finally, Watson and Pollack [2001] used a collection of simple number games to illustrate three important coevolutionary limitations, namely, focusing, which is a parallel to GAN mode collapse, relativism, which is a parallel to GAN discriminator collapse, and loss of gradients, which is a parallel to GAN vanishing gradients.

A general reason offered for these limitations is that, despite the algorithm's stochasticity, the populations lack sufficient solution diversity to disrupt premature convergence in the form of an oscillation or move the search away from an undesired equilibria. Essentially, the population should serve as a source of novelty and a genotypically or phenotypically converged population fails in this respect. One method of preventing convergence is to supplement one or both populations with 
a memory that holds genotypes that have been previously visited. Memory has been implemented as a hall-of-fame [Nolfi and Floreano 1998] or Pareto archive [Popovici et al. 2012]. Solutions in the memory also serve as reference points for measuring the relative quality of a current solution and detecting a diminishing gradient. Solution diversity has also been explicitly improved with competitive fitness sharing [Rosin and Belew 1997], separation, e.g., a spatial topology [Mitchell 2006], or a spatial topology and temporal segregation [Harper 2014].

A spatial (2D toroidal) topology is an effective means of controlling the mixing of adversarial populations in coevolutionary algorithms [Mitchell 2006]. In a conventional setup, the members of populations are divided up on a grid of cells and overlapping neighborhoods for each cell are identified (see Figure 1). A neighborhood is defined by the cell itself and its adjacent cells and specified by its size, $s$. Coevolution proceeds at each cell with sub-populations drawn from the neighborhood. Compared to an algorithm where all members can interact with each other, this reduces the cost of interaction from $O\left(N^{2}\right)$ to $O(N s)$, where $N$ is the size of each population. A von Neumann neighborhood with $s=5$ cells (center and four cells, respectively, North, East, South, and West) is commonly used [Husbands 1994] (see Figure 1). With this design, each neighborhood can evolve in semi-isolation as the search space is explored [Mitchell 2006; Williams and Mitchell 2005]. It is this approach: spatial separation via a grid, that Lipizzaner applies to GAN training.

\subsection{Evolutionary Computation, Neural Nets, and GANs}

Evolutionary Computation has been used to design neural networks through neuroevolution [Camero et al. 2020; Stanley et al. 2019]. E.g., NEAT and HYPER-NEAT [Stanley et al. 2019; Stanley and Miikkulainen 2002] have been used to evolve network architectures [Costa et al. 2019]. In addition, population-based training of neural networks [Jaderberg et al. 2017] has been investigated. Moreover, a combination of elements from deep learning and artificial life demonstrated that coevolutionary neural population models can simulate population dynamics and that evolutionary game theory can describe the behavior [Moran and Pollack 2018].

Without explicit remedies, competitive coevolutionary algorithms experience what we call pathologies or limitations in the form of non-convergent behavior, i.e., oscillations, and undesired but stable equilibrium. As such, they share similarities with GAN training. The GAN training can also be seen as a two-player game-one that is solved using gradient-based updates to simultaneously optimize a minimax objective. Commonly observed GAN training pathologies are mode collapse [Arora et al. 2018] (the generator can only generate one mode of the distribution), discriminator collapse [Li et al. 2017b] (the discriminator cannot discriminate any modes), as well as vanishing gradients [Arjovsky and Bottou 2017] (there are no training gradients, i.e., one adversary is too strong/weak).

One example of combining evolutionary computation and GANs is E-GAN [Wang et al. 2019]. It evolves a population of three independent loss functions defined according to three metrics (Jenson-Shannon Divergence (JSD), Least Squares (LS) distance, and a combination of JSD and Kullback-Leibler (KL) divergence) [Wang et al. 2019]. Each loss function is independently used to train a generator from some starting condition over a batch of training data. The generators produced by the loss functions are evaluated by a single discriminator that returns a fitness value for each generator. The best generator is then selected and training continues, with the next training batch, and the three different loss functions. The use of different loss functions overcomes the limitations of a single individual training objective and better adapts the population to the evolution of the discriminator. The results for problems in the image domain have shown that E-GAN is able to obtain higher (better) inception scores, compared to a canonical GAN baseline, while showing comparable stability when it converges. E-GAN relies on the evolutionary population to introduce diversity into the training by its different loss functions and it uses what can be described 
as evolutionary selection when updating the best generator of a batch. A second example, leading to Lipizzaner, is described in Al-Dujaili et al. [2018a]; Schmiedlechner [2018]. This approach was a competitive coevolutionary algorithm but it did not include the spatial distribution of the population. Interestingly, it included a variation that even evolved (with CMA-ES) the network parameters, though this variant was not used for Lipizzaner due to its inefficiency over using the gradient information that is readily available.

\subsection{Scaling Evolutionary Computing for Machine Learning}

There are multiple examples of scalable and large-scale evolutionary computation systems. One is EC-Star [Hodjat et al. 2014], which runs on hundreds of desktop machines. In another, one team [Salimans et al. 2017] applied a simplified version of Natural Evolution Strategies [Wierstra et al. 2008] with a novel communication strategy to a collection of reinforcement learning benchmark problems. Due to better parallelization over thousand cores, they achieved fast training times (wall-clock time). They ran their experiments on a computing cluster of 80 machines and 1,440 CPU cores [Salimans et al. 2017]. Another team [Stanley and Clune 2017] showed that deep convolutional networks with over $4 \mathrm{M}$ parameters trained with genetic algorithms can also reach competitive results. They employed a range of hundreds to thousands of CPU cores (depending on availability). Deep networks have also been evolved on HPC [Young et al. 2017].

The bulk of attempts to improve GAN training has been designed to run on a single machine (or a single GPU). The advent of large-scale parallel computation infrastructure with evolutionary computation enables us to scale GAN training to spatial grids.

\subsection{Training GANs}

Robust GAN training is still an open research topic [Arora et al. 2017]. Simple theoretical models have been proposed to provide a better understanding of the problem [Li et al. 2017b]. These were investigated in Al-Dujaili et al. [2018a], which describes preliminary work that establishes that an evolutionary algorithm can ameliorate specific training problems. This work formed the basis of Lipizzaner. Coevolution is one approach among many, none offering a complete resolution. One example is hard-coded conditions to decrease the optimizers' learning rate after a given number of iterations [Radford et al. 2015]. Several heuristic practices have been suggested to stabilize the training [Chintala et al. 2016]. There are also ensemble approaches and approaches that change the generator's or discriminator's cost function.

Heuristic Approaches. Goodfellow in his 2014 paper describes basic GAN training [Goodfellow et al. 2014]. Some training aspects can be modified, e.g., flipping a maximization objective to a minimization objective to obtain a more informative gradient. See Brownlee [2019] for more details.

Ensemble Approaches. The use of multiple generators and/or discriminators for improving robustness has also been studied. This includes: training a cascade of GANs [Wang et al. 2016]; sequentially training and adding new generators with boosting techniques [Tolstikhin et al. 2017]; training multiple generators and discriminators in parallel [Jiwoong Im et al. 2016]; training an array of discriminators specialized in a different low-dimensional projection of the data [Neyshabur et al. 2017]; using several adversarial "local" pairs of networks that are trained independently so a "global" supervising pair of networks can be trained against them [Chavdarova and Fleuret 2018]; and learning from a dynamic ensemble of discriminators [Mordido et al. 2018]. Lipizzaner also takes an ensemble approach. It is distinctive in evolving ensembles at a local (neighborhood) level, co-tuning the ensemble weights, and supporting the choice of the best ensemble (among all neighborhoods).

Cost Function Options. Cost function approaches change the original Jensen-Shannon divergence (JSD) loss function [Goodfellow et al. 2014] to alternative functions in an effort to address 
poor GAN training. There are multiple studies of alternate functions, including, e.g., Borji [2019], which includes a large table of GAN evaluation measures and Lucic et al. [2018] and Shmelkov et al. [2018], which use two measures that approximate the recall (diversity) and precision (quality of the image). Arjovsky et al. [2017] introduces the popular Wasserstein loss that we include in our empirical comparison studies. There are also domain-specific measures outside the image domain, e.g., for text probability-based language model metrics Tevet et al. [2018]. Other cost function approaches include Mao et al. [2017], Nguyen et al. [2017], and Zhao et al. [2016]. No single loss function is a solution for all problems.

Lipizzaner incorporates the above approaches in its evolutionary GAN training. We next present our notation and formalize the goal of GAN training.

\section{GOAL OF GAN TRAINING AND NOTATION}

We adopt notation similar to Arora et al. [2017] and Li et al. [2017b] (for an overview of notation, see Table 1). Let $\mathcal{G}=\left\{G_{g}, g \in \mathbb{G}\right\}, \mathbb{G} \subset \mathbb{R}^{p}$ and $\mathcal{D}=\left\{D_{d}, d \in \mathbb{D}\right\}, \mathbb{D} \subset \mathbb{R}^{p}$ denote sets of generators and discriminators. $G_{g}$ is a function $G_{g}: \mathbb{R}^{l} \rightarrow \mathbb{R}^{v}$ parameterized by $g$. $D_{d}$ is a function $D_{d}: \mathbb{R}^{v} \rightarrow$ $[0,1]$ parameterized by $d$. The generator $G_{g}$ defines a distribution $\mathcal{T}_{G_{g}}$; this is done by generating $z$ from an $l$-dimensional Gaussian distribution and then applying $G_{g}$ on $z$ to generate a sample $x=G_{g}(z)$ of the distribution $G_{g}$. Finally, let $\mathcal{T}_{*}$ be the unknown target distribution to which we would like to fit our generative model $G_{g}$.

Formally, the goal of GAN training is to find parameters $g$ and $d$ to optimize the objective function

$$
\begin{gathered}
\min _{g \in \mathbb{G}} \max _{d \in \mathbb{D}} \mathcal{L}(g, d), \text { where } \\
\mathcal{L}(g, d)=\mathbb{E}_{x \sim \mathcal{T}_{*}}\left[\varphi\left(D_{d}(x)\right)\right]+\mathbb{E}_{x \sim \mathcal{T}_{G_{g}}}\left[\varphi\left(1-D_{d}(x)\right)\right],
\end{gathered}
$$

and $\varphi:[0,1] \rightarrow \mathbb{R}$, is a concave measuring function. In practice, we have access to a finite number of training samples $x_{1}, \ldots, x_{v}$ to approximate $\mathcal{D}_{G_{g}}$ or $G_{g}$. Therefore, an empirical version $\frac{1}{v} \sum_{i=1}^{v} \varphi\left(D_{d}\left(x_{i}\right)\right)$ is used to estimate $\mathbb{E}_{x \sim \mathcal{T}_{*}}\left[\varphi\left(D_{d}(x)\right)\right]$.

One way of expressing Lipizzaner is as a function $F$ that returns an ensemble of generator parameters and their ensemble weights:

$$
\mathrm{e}=F(\mathrm{X}, \mathrm{y}), F: \mathcal{X} \times \mathcal{Y} \rightarrow \mathcal{E}
$$

The variables that are optimized are $\mathrm{y}=[\mathrm{g}, \delta, \mathrm{w}], \mathrm{y} \in \mathcal{Y}, \mathcal{Y}=\left\{\mathbb{G}^{s} \times \mathbb{R} \times \mathbb{R}^{s}\right\}$, the ensemble size is $s, s \in \mathbb{N}$, the parameters of each generator $G_{g}$ in the ensemble $\mathbf{g}=\left[G_{g_{1}}, \ldots, G_{g_{s}}\right]$, the learning rate for neural network training $\delta, \delta \in \mathbb{R}$ and the ensemble mixture weights $\mathbf{w}, \mathbf{w} \in \mathbb{R}^{s}$, and $\mathrm{X}$ is a dataset (a sample from the target distribution $\mathcal{T}_{*}$ ). We focus on finding the best ensemble of generators $\mathbf{e}=[\mathrm{g}, \mathbf{w}], \mathbf{e} \in \mathcal{E}, \mathcal{E}=\left\{\mathbb{G}^{s} \times \mathbb{R}^{s}\right\}$ according to a measuring function $h: \mathbb{G}^{s} \times \mathbb{R}^{s} \rightarrow \mathbb{R}$. This can be stated as:

$$
\underset{\mathbf{y} \in \mathbf{y}}{\arg \max } h(F(\mathbf{X}, \mathbf{y} \mid \Theta))
$$

by using the GAN training Equation (1) and $\Theta$ is the set of parameters $\Theta=\left\{m, s, M, T, \tau, \beta, \mu, \mathbf{w}, \delta, G_{g}, D_{d}, f_{s}^{\min }\right\}$, shown in Table 1 .

Lipizzaner uses a square toroidal grid with $N$ cells, $N=m \times m$. A cell $n, n=[g, d, \delta, \mathbf{w}], n \in$ $\mathbf{N}, \mathbf{N}=\left\{\mathbb{R} \times \mathbb{R}^{s} \times \mathbb{G} \times \mathbb{D}\right\}$ has a neighborhood $\left.\mathbf{n}=\left\{n_{1}, \ldots, n_{s}\right\}, n_{i} \in \mathbf{N}\right\}$ that allows it to form two sub-populations of models: $\mathbf{g}$ and $\mathbf{d}$. We denote the size of this neighborhood by $s$ and the number of neighborhoods is $N$. We use a five-cell von Neumann neighborhood $(s=5)$ (see Figure 1).

For the $k$ th neighborhood in the grid $\mathbf{n}_{k}, 1 \leq k \leq N$, we refer to the generator in its center cell by $g_{k, 1}$ and the generators in the rest of the neighborhood by $g_{k, 2}, \ldots, g_{k, s}$, respectively. 
Furthermore, we denote the union of these generators for the $k$ th generator neighborhood by $\mathbf{g}_{k}=\cup_{i=1}^{s}\left\{\mathbf{g}_{k, i}\right\} \subseteq\left\{g_{1,1}, \ldots, g_{N, 1}\right\}$.

In Lipizzaner, we use generators of the $N$ sub populations each of size $s$ to form $N$ ensembles, and the best ensemble is returned by Lipizzaner. For the ensembles of generators $g$ the $s$-dimensional ensemble mixture weight vector $\mathbf{w}$ is defined as follows:

$$
\mathbf{e}^{*}=\underset{\mathbf{g}_{k}, \mathbf{w}_{k}: 1 \leq k \leq N}{\arg \max } \sum_{i=1}^{s} h\left(g_{k, i}, w_{k, i}\right),
$$

where $w_{k, i}$ represents the probability that a data point comes from the $i$ th generator in the neighborhood, with $\sum_{i=1}^{s} w_{k, i}=1$.

GAN training. Multiple loss functions $\phi$ have been used for GAN training. The ones we are concerned with are (written for generators):

(1) Binary cross entropy (BCE) loss, where the model's objective is to minimize the JensenShannon divergence (JSD) between the real $(p)$ and fake $(q)$ data distributions, i.e., $J S D(p \| q)$.

$$
M_{G}^{B C E}=\frac{1}{2} \mathbb{E}_{x \sim G_{g}}\left[\log \left(1-D_{d}(x)\right)\right]
$$

(2) Mean squared error (MSE) loss, where the model's objective is to minimize the average of the squares of the errors, e.g., Mao et al. [2017]:

$$
M_{G}^{M S E}=\mathbb{E}_{x \sim G_{g}}\left[\log \left(D_{d}(x)-1\right)^{2}\right] .
$$

(3) Heuristic loss (HEU) objective maximizes the probability of the discriminator being mistaken by minimizing the objective function [Wang et al. 2019]:

$$
M_{G}^{H E U}=\frac{1}{2} \mathbb{E}_{x \sim G_{g}}\left[\log \left(D_{d}(x)\right)\right]
$$

This objective is equal to minimizing $[K L(p \| q)-2 J S D(p \| q)]$.

(4) Wasserstein (WASS) loss. Wasserstein GANs [Arjovsky et al. 2017] minimize the Wasserstein Distance between the two probability distributions. However, it is practically intractable. Thus, this method optimizes WASS loss objective

$$
M_{G}^{W A S S}=\mathbb{E}_{x \sim G_{g}}\left[f_{w}(x)\right]
$$

where $f_{w}$ comes from a family of K-Lipschitz continuous functions parameterized by $w$ [Arjovsky et al. 2017].

With these formalities in place, in the next section, we describe Lipizzaner.

\section{THE LIPIZZANER FRAMEWORK}

In this section, we describe the framework we call Lipizzaner for improved GAN training by promoting diversity. Lipizzaner uses spatial distribution to address the quadratic computational complexity of the basic competitive coevolutionary algorithm's adversarial competitions. It also includes:

- gradient-based learning to update the neural networks' parameters of the generator $g$, discriminator $d$, and learning rate $\delta$,

- Gaussian-based mutations to update the learning rate and weight ensemble parameters $\delta, w$,

- evolutionary selection and replacement, and

- overlapping cells $n_{i}$ between neighborhoods $\mathbf{n}_{i}, \mathbf{n}_{j}$, with sub-populations of generators $\mathbf{g}$ and discriminators $\mathbf{d}$ in the neighborhoods. 
Table 1. Names Used for Lipizzaner

\begin{tabular}{|c|c|}
\hline Name & Constants \\
\hline C & GAN training cost, used for computational complexity analysis, $C \in \mathbb{Z}$ \\
\hline \multirow{2}{*}{$\begin{array}{l}s_{n} \\
f_{s}^{\min }\end{array}$} & Maximum neighborhood size, $s_{n}=5$ (a von Neumann neighborhood) \\
\hline & Solution concept for selection and replacement that uses the minimum value ("best worst case") \\
\hline \multicolumn{2}{|r|}{ Randomly Initialized and Evolved GAN Variables } \\
\hline$\delta$ & Learning rate of a neural network (generator/discriminator), a parameter, $\delta \in[0, \ldots, 1]$ \\
\hline w & Ensemble mixture weights of the generator ensemble mixture, a parameter, $\mathbf{w} \in[0, \ldots, 1]^{s}$ \\
\hline \multicolumn{2}{|r|}{ Randomly Initialized and SGD-trained GAN Variables } \\
\hline$g$ & Parameters of the generator, $g \in \mathbb{G}$. \\
\hline$d$ & Parameters of the discriminator, $d \in \mathbb{D}$. \\
\hline \multicolumn{2}{|r|}{ Configuration Parameters for Spatial Coevolution } \\
\hline$m$ & The grid size, $m \in \mathbb{Z}$ \\
\hline$s$ & Neighborhood size, derived from the cardinality of the neighborhood $s=\min \left(m^{2}, s_{n}\right)$ \\
\hline$M$ & Loss functions, $M \in \mathcal{M}$ \\
\hline$T$ & Number of generations (epochs), $T \in \mathbb{Z}$ \\
\hline$\tau$ & Tournament size, $\tau \in \mathbb{Z}$ \\
\hline$\beta$ & Mutation probability for learning rate, $\beta \in \mathbb{R}$ \\
\hline$\mu$ & Mutation probability for mixture weights, $\mu \in \mathbb{R}$ \\
\hline$G_{g}$ & A function parameterized by $g, G_{g}: \mathbb{R}^{l} \rightarrow \mathbb{R}^{v}$ \\
\hline$D_{d}$ & A function parameterized by $d, D_{d}: \mathbb{R}^{v} \rightarrow[0,1]$ \\
\hline $\mathrm{X}$ & Data set sample, $\mathrm{X} \in X$ \\
\hline$\Theta$ & Parameters for Lipizzaner $\Theta=\left[m, s, M, T, \tau, \beta, \mu, \mathbf{w}, \delta, G_{g}, D_{d}, f_{s}^{\mathrm{min}}\right]$ \\
\hline \multicolumn{2}{|r|}{ GAN Notation } \\
\hline$\varphi$ & Concave measuring function, $\varphi:[0,1] \rightarrow \mathbb{R}$ \\
\hline $\mathcal{G}$ & Set of generators, $\left\{G_{g}, g \in \mathbb{G}\right\}, \mathbb{G} \subset \mathbb{R}^{p}$ \\
\hline $\mathcal{D}$ & Set of discriminators, $\left\{D_{d}, d \in \mathbb{D}\right\}, \mathbb{D} \subset \mathbb{R}^{p}$ \\
\hline$l$ & Dimension of Gaussian distribution that generates generator input, $l \in \mathbb{Z}$ \\
\hline$z$ & Generator input dimension, $z \in \mathbb{Z}$ \\
\hline$v$ & Generator output dimension, this is also the discriminator input dimension, $v \in \mathbb{Z}$ \\
\hline $\mathcal{T}_{G_{g}}$ & Distribution defined by the generator, $G_{g}$ \\
\hline $\mathcal{T}_{*}^{3}$ & Target distribution for the generator, $G_{g}$ \\
\hline & Dataset size, $e=|\mathrm{X}|$ \\
\hline $\mathcal{L}(g, d)$ & $\begin{array}{l}\text { Gan training objective function (see Equation (1)). Used to calculate fitness for GAN coevolution } \\
\text { (see Algorithm 3). }\end{array}$ \\
\hline \multicolumn{2}{|r|}{ Spatial Coevolution GAN Notation } \\
\hline$\phi$ & Fitness of a GAN (see Algorithm 4), $\phi_{g}$ for generator, and $\phi_{d}$ for discriminator \\
\hline$n$ & A cell in the grid, $n=[g, d, \delta, \mathrm{w}], n \in \mathrm{N}, \mathrm{N}=\left\{\mathbb{G} \times \mathbb{D} \times \mathbb{R} \times \mathbb{R}^{s}\right\}$ \\
\hline$n_{k}$ & The $k$ th cell, $1 \leq k \leq N$ \\
\hline $\mathrm{n}$ & The neighborhood, contains $\left[n_{1}, \ldots, n_{s}\right], \mathbf{n} \in \mathrm{N}^{s}$, these are the sub-populations \\
\hline $\mathbf{n}_{k}$ & The $k$ th neighborhood, $\mathbf{n}_{k} \in \mathrm{N}$ \\
\hline$g_{k, 1}$ & Generator at the center cell of the $k$ th neighborhood, $g_{k} \in \mathrm{g}, 1 \leq k \leq N$ \\
\hline$N$ & Number of cells, $N=m^{2}$ (also the population size) \\
\hline $\mathrm{N}$ & Grid population $\mathbf{N}=\left[\mathbf{n}_{1}, \ldots, \mathbf{n}_{N}\right]$ \\
\hline g & The sub-population of generators $\mathrm{g}=\left[g_{1}, \ldots, g_{s}\right]$ in a neighborhood \\
\hline d & The sub-population discriminators $\mathbf{d}=\left[d_{1}, \ldots, d_{s}\right]$ in a neighborhood \\
\hline$F$ & Lipizzaner function (see Equation (2)), $F: X \times \mathbb{G}^{s} \times \mathbb{R} \times \mathbb{R}^{s} \rightarrow \mathbb{G}^{s} \times \mathbb{R}^{s}$ \\
\hline$h$ & Generator measuring function, $h: \mathbb{G}^{s} \times \mathbb{R}^{s} \rightarrow \mathbb{R}, q=h(\mathrm{~g}, \mathbf{w})$ (here FID) \\
\hline$q$ & $\begin{array}{l}\text { Generator ensemble quality score } q=h(\mathrm{~g}, \mathrm{w}) \text {, here FID score. Used as fitness for weight evolution } \\
\text { (see Algorithm 2) }\end{array}$ \\
\hline y & Variables optimized by Lipizzaner, $\mathrm{y}=[\mathrm{g}, \delta, \mathrm{w}], \mathrm{y} \in \mathcal{Y}, \mathcal{Y}=\left\{\mathbb{G}^{s} \times \mathbb{R} \times \mathbb{R}^{s}\right\}$ \\
\hline e & Ensemble of generators, $\mathrm{e}=[\mathrm{g}, \mathrm{w}], \mathrm{e} \in \mathcal{E}, \mathcal{E}=\left\{\mathbb{G}^{s} \times \mathbb{R}^{s}\right\}$ \\
\hline $\mathrm{e}^{*}$ & Best ensemble of generators, $\mathbf{e}=[\mathbf{g}, \mathbf{w}]$ (see Equation (4)) \\
\hline
\end{tabular}

Parameters, variables and constants are shown. Variables are optimized, parameters also include the initial variable values in Lipizzaner, and constants are used for the computational complexity analysis. 


\begin{tabular}{|c|c|c|c|}
\hline \multicolumn{4}{|c|}{ Toroidal Grid } \\
\hline 0 & 1 & 2 & 3 \\
\hline 4 & 5 & 6 & 7 \\
\hline 8 & 9 & 10 & 11 \\
\hline 12 & 13 & 14 & 15 \\
\hline
\end{tabular}

$\mathrm{N}=4 \times 4, \mathrm{~m}=4$
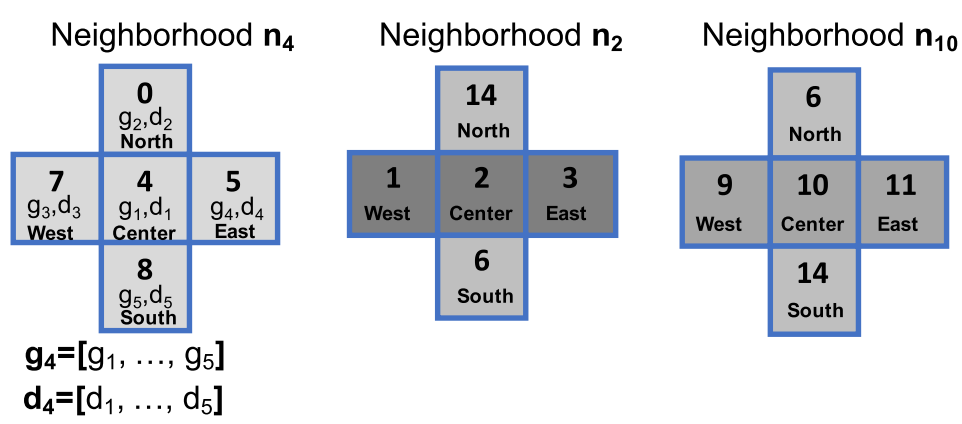

Fig. 1. Illustration of overlapping von Neumann neighborhoods on a toroidal $4 \times 4$ grid, color indicates three different neighborhoods and similar color in same neighborhood indicates overlap. Note how a cell update at $n_{4}$ can be communicated to $\mathbf{n}_{5}$ when $n_{5}$ gathers its neighbors. If $\mathbf{n}_{5}$ is then updated with the updated value from $n_{4}$, then the value has propagated. The $n_{6}$ value is in the range of both $\mathbf{n}_{2}$ and $\mathbf{n}_{10}$. Propagation runs laterally and vertically. We also show an example of a cell's generator $\mathbf{g}$ and discriminator $\mathbf{d}$ sub-populations (based on its neighborhood) for $\mathbf{n}_{4}$.

First, we walk the reader through Lipizzaner considering a specific use case. Then, we more formally describe its algorithms. Finally, we highlight Lipizzaner's different means of promoting diversity.

\subsection{High-level Description with Example Use Case}

First, consider a population $N=m^{2}$, for some grid $m^{2}$, where each population member contains four units of information, generator weights $g$, discriminator weights $d$, learning rate $\delta$, weight mixture $w$, to be evolved. Because the generator $G_{g}$ and discriminator $D_{d}$ are adversarial, they could be split into two populations, each of size $N$ to coevolve. The fitness $\phi_{g}$ of a generator would be its maximum loss over all discriminators, and discriminator's fitness $\phi_{d}$ would be its maximum over all generators. The cost of this coevolutionary setup, i.e., evaluating all generators vs. all discriminators has complexity $O\left(N^{2}\right)$. Instead of this approach, to reduce the complexity to $O\left(N s^{2}\right)$, Lipizzaner works with $N$ sub-populations of size $s=5$, which are each a member, centered on a neighborhood $\mathbf{n}_{k}$ positioned in a 2D grid (Center $\mathbf{n}_{k, 1}$ ), and the member's spatial neighbors to the North, East, West, and South $\mathbf{n}_{k, 2}, \ldots, \mathbf{n}_{k, 5}$. In this approach, each sub-population $\mathbf{n}$ is then divided into two populations: five generators $\mathbf{g}_{\mathbf{k}}$ and five discriminators $\mathbf{d}_{\mathbf{k}}$. These are co-evolved.

We now describe in more detail the use of Lipizzaner to model an image dataset. Lipizzaner is set up with a $N=m^{2}, m=4,4 \times 4,2$-dimensional toroidal grid where, per the prior high-level description, each cell $n$ contains the learning rate $\delta$ during GAN training, five weights $\mathbf{w}$ for its ensemble mixture of solutions, a generator $g$, and discriminator $d$ (see Figure 1).

In parallel and asynchronously (see Figure 2), each cell $n$ executes the same generational algorithm that is illustrated in Figure 3. Given each cell $n$ has its neighborhood $\mathbf{n}$ with sub-populations of generators $\mathbf{g}$ and discriminators $\mathbf{d}$, any generator (discriminator) can be trained pairwise with any discriminator (generator) via SGD. A generator's quality in the GAN training context is derived from its pairwise performance (i.e., loss given by $\mathcal{L}$ ) against all discriminators in the sub-population (neighborhood) $\mathbf{n}$. Pairwise performance is measured by using the generator to generate a number of images and summing its errors with respect to its paired discriminator detecting its images as "real" (good for the generator, bad for the discriminator) or "fake" (good for 


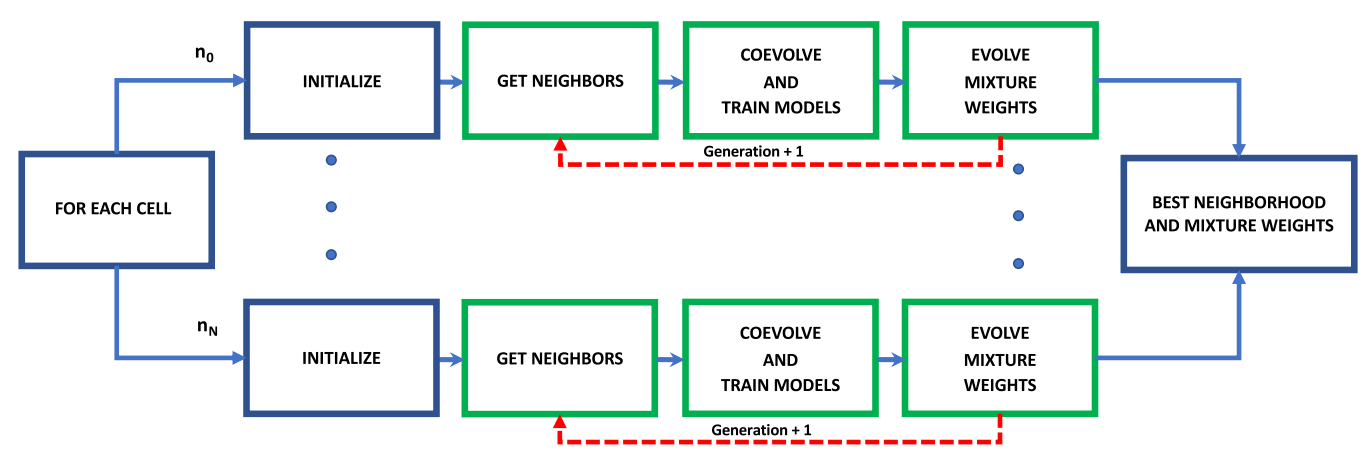

Fig. 2. Flowchart of Algorithm 1::Lipizzaner (the different colored boxes are elaborated in Figure 3). Each cell neighborhood executes in parallel, asynchronously. Each cell neighborhood then iterates over a number of generations. At the beginning of a generation neighbors are collected, while at the end the cell itself is updated (see line 4 of Algorithm 1::Lipizzaner and line 17 of Algorithm 3::CoevolveAndTrainModels). Information propagation is facilited by each cell updating itself with the best of its neighborhood (see Algorithm 3::CoevolveAndTrainModels line 17).

the discriminator, bad for the generator). The discriminator's performance $(\mathcal{L})$ is how frequently it correctly detects a "fake" and a "real" image.

Within the cell's generational algorithm, generator ensemble weights $\mathbf{w}$ are selected (and replicated) based on FID score. This is an image-specific estimate of "absolute" generative model quality [Heusel et al. 2017a]. GAN training then proceeds by mini-batch. For each mini-batch, first, a random discriminator is drawn from the sub-population $\mathbf{d}$. All generators $\mathrm{g}$ are trained pairwise with this discriminator. Here, to promote diversity, the models are pairwise randomly assigned a loss function to use during the GAN training. Any or some or all of three loss functions (with corresponding minimization objectives w.r.t. the generator) are used in the image use case: binary cross-entropy, least square, and a KL divergence-based heuristic [Wang et al. 2019] (see Section 3). Then, one of the (trained) generators is randomly drawn and all discriminators $\mathbf{d}$ are trained pairwise with it. All GAN training is done with stochastic gradient descent (SGD) based upon loss $\mathcal{L}$ calculated with the model's randomly assigned loss function.

After multiple mini-batches, fitness $\phi$ (based on loss $\mathcal{L}$ ) is once again evaluated so the discriminator and generator of the now-trained sub-population with the lowest loss (best) replace those of the cell center $n_{k, 1}$. This is an asynchronous operation. Next, this sub-population $\mathbf{n}$ is considered collectively. Lipizzaner evolves ensemble "mixture weights" w for each of the five generators $\mathrm{g}$ in the sub-population using random perturbation and keeping the perturbation if there is an improvement in the FID score, also known as 1+1 Evolutionary Strategy (ES) [Loshchilov 2013, Algorithm 2.1]. The ensemble mixture weights $\mathbf{w}$ are normalized and represent the likelihood a generator will be used to generate an image. The evolution of the mixture weights $\mathbf{w}$ will reflect the diminished value of badly performing generators and the increased value of good ones. The $1+1$ ES measures the fitness of a set of mixture weights with FID score on the image dataset.

After some number of generations $T$ or when a time limit is reached, evolution within each cell $n$ halts. Every cell neighborhood $\mathbf{n}$ is then evaluated as a weighted mixture to identify the best neighborhood $\mathbf{e}^{*}$ of the grid. For this evaluation, each mixture weight $w$ is treated as a probability of using one generator $g$. The mixture generates multiple (fake) sample images that are used to calculate an FID score $q$ reflecting the quality of the sampled images. This best ensemble mixture $\mathrm{e}^{*}$ is Lipizzaner's answer to the generative modeling problem.

We now proceed to a more detailed description that references pseudocode and flowcharts. 


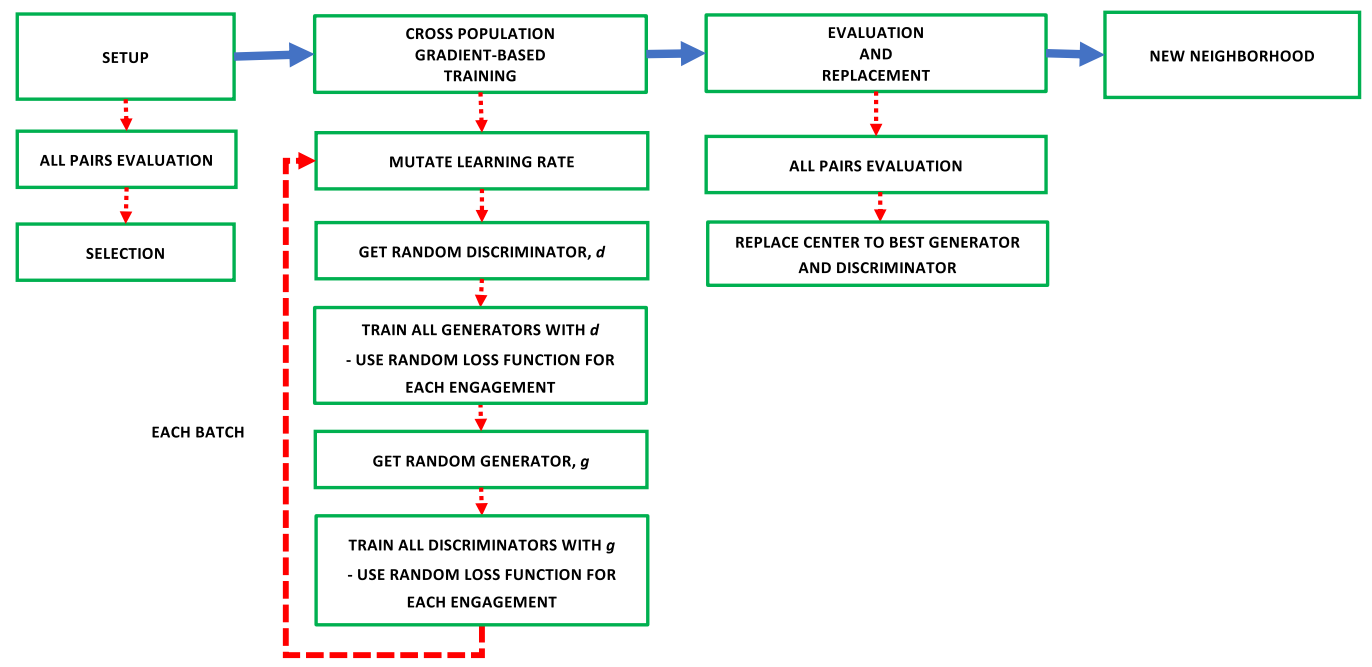

Fig. 3. Flowchart of Algorithm 3::Lipizzaner (expansion of the different colored boxes in Figure 2). This cellcentered algorithm is called each generation $t$, after generator and discriminator neighbors from adjoining cells are gathered. At each generation $t$, it performs evolutionary selection and replacement on generators $\mathrm{g}$ and discriminators $\mathrm{d}$ that are improved with SGD. Note also the use of random loss function assignment to promote diversity and the mutation of the training learning rate $\delta$. "Best" is based on a solution concept $f_{s}^{\text {min }}$, the "best worst case" loss, each model being assigned this based on the loss over all its adversarial models.

\subsection{Algorithmic Description of Lipizzaner}

Lipizzaner starts with Algorithm 1::Lipizzaner, which immediately starts parallel execution on each cell $n$. Each cell neighborhood $\mathbf{n}$ randomly initializes single discriminator $d$ and generator $g$ neural network models (for readability, we just refer to these as models when discriminators and generators are interchangeable). Thus, two global (grid-scale) adversarial populations emerge, $\left[g_{1}, \ldots, g_{N}\right]$ a population of generators and $\left[d_{1}, \ldots, d_{N}\right]$ a population of discriminators, where the grid size $N$ is the global population size. These populations are one source of diversity in Lipizzaner, and we refer to this diversity as occurring in genome space. Each cell $n$ also initializes its parameters-learning rate $\delta$ and mixture weights $\mathbf{w}$.

Next, we describe the steps in Lipizzaner in more detail.

Evolving Generator Mixture Weights. Lipizzaner at the highest level searches for and returns a mixture of generators e composed from a neighborhood $\mathbf{n}$. It evolves a mixture weight vector w for each neighborhood $\mathbf{n}$ using an ES-(1+1) algorithm that optimizes for generator ensemble performance $q$, here FID.

Generation Loop. Algorithm 1::Lipizzaner directs each cell executing in parallel to iterate over a generational loop. Each generation $t$, a cell, $n_{k}$, starts by copying the latest versions of its neighbors $\mathbf{n}_{k}$ to set up its sub-populations $\mathbf{g}_{k}$ and $\mathbf{d}_{k}$. Each cell next independently executes Algorithm 3::CoevolveAndTrainModels. This algorithm terminates with the cell neighborhood updating its own models, i.e., $\mathbf{g}_{k, 1}, \mathbf{d}_{k, 1}$. These are two key steps that facilitate information propagation-at the beginning of a generation $t$ a cell $n$ creates a sub-population $\mathbf{n}$ by reading the latest copies of models from its neighbors in the grid, coevolution is applied within the 
ALGORITHM 1: Lipizzaner: In parallel, for each cell, initialize settings then iterate over each generation $t$. Each generation $t$, retrieve neighbors to build generator $\mathbf{g}_{k}$ and discriminator $\mathbf{d}_{k}$ subpopulations, evolve generators $\mathbf{g}_{k}$ and discriminators $\mathbf{d}_{k}$ trained with SGD, replace self $n_{k, 1}$ with best, and finally evolve weights $\mathbf{w}$ for a neighborhood mixture model $\mathbf{e}$.

Input: $\mathrm{T}$ : Total generations, $\mathbf{N}$ : Population on grid cells, $s:$ Neighborhood size, $\theta_{E A}:$ Parameters for MixtureEA, $\theta_{C O E V}$ : Parameters for CoevolveAndTrainModels

Return: e : ensemble of generators and mixture weights
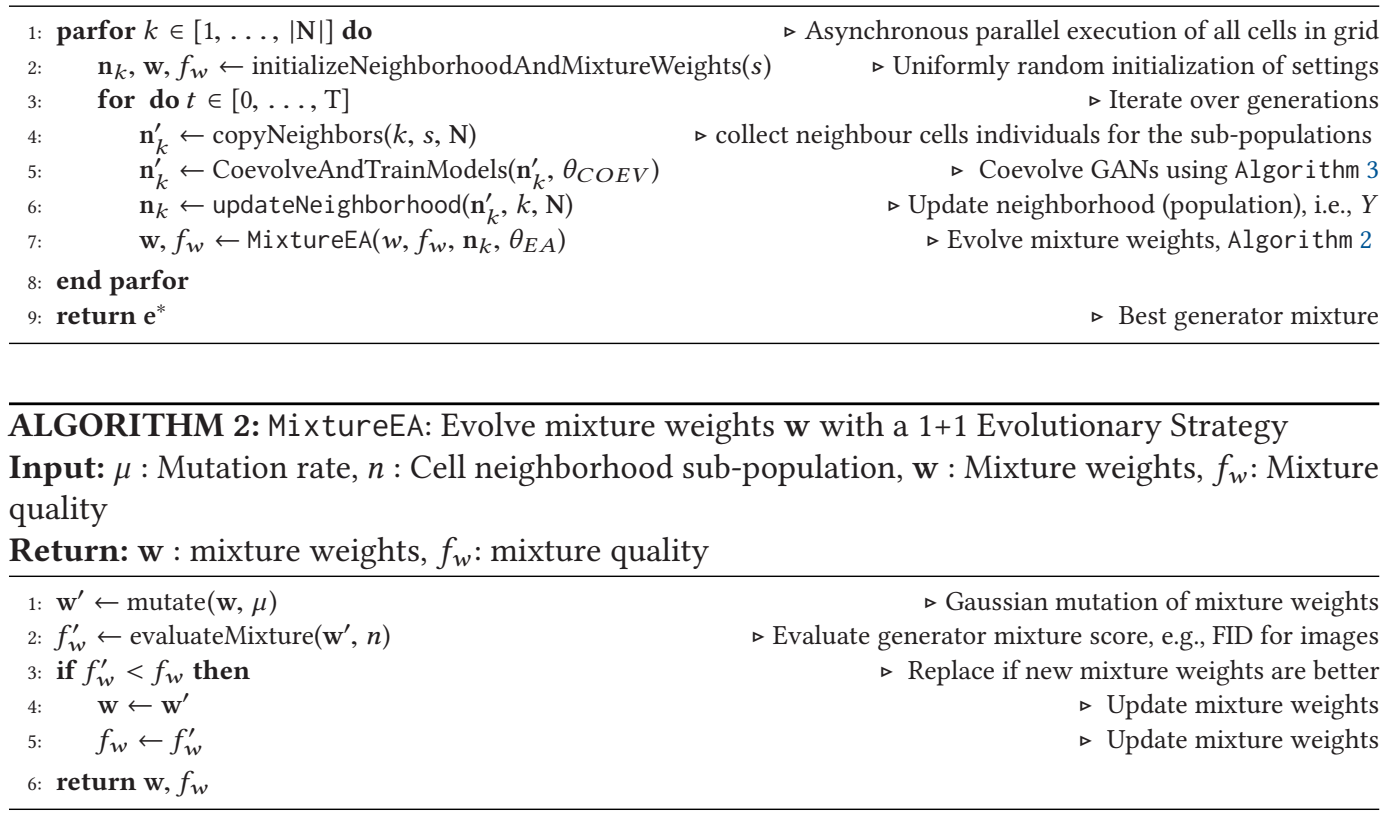

sub-population, and the best generator and discriminator are then stored back into the cell in the grid, ready to be copied by other neighborhoods.

Upon each cell returning its sub-populations to Algor ithm 1::Lipizzaner, the mixture weights $\mathbf{w}$ are evolved (as shown in previous paragraph). After all generations are completed (or a computational deadline is reached), Algor i thm 1::Lipizzaner then selects the best performing ensemble $\mathrm{e}^{*}$ across the entire grid (see following paragraph) and returns it as Lipizzaner's solution.

Selection and Replacement. Selection promotes fitter models over less fit ones when updating a sub-population n. Lipizzaner uses tournament selection, Algorithm 3::CoevolveAndTrainModels line 3. First, the latest neigbors cell models are copied to form the sub-populations and then Lipizzaner applies selection. After all GAN training is completed and all models are evaluated again, based on the training loss $\mathcal{L}$ the least fit generator and discriminator in the sub-populations are replaced with the fittest ones, Algorithm 3::CoevolveAndTrainModels line 17.

Fitness Evaluation. Integral to the sub-populations approach in Lipizzaner is the notion that a model's performance $\mathcal{L}$ depends on its adversary (see Algorithm 4::EvaluateGANPairs). Calculated with some loss function $M$, performance can be expected to vary. To accommodate this, in Lipizzaner, fitness $\phi$ of a model $\left(g_{i} \in \mathrm{g}\right.$ or $\left.d_{j} \in \mathbf{d}\right)$ is based on a solution concept [Popovici et al. 2012], $f_{s}^{\min }$, that is the "best worst case" loss $\mathcal{L}$ against all its adversaries. 
ALGORITHM 3: CoevolveAndTrainModels: Select a new sub-population from the current one. Assign all models a loss function at random. Each mini-batch trains discriminators $\mathbf{d}$ against a randomly drawn generator and generators $\mathrm{g}$ against a randomly drawn discriminator, using SGD. Evaluate all against each other, using the solution concept, $f_{s}^{\min }$, of "best worst case" loss value to choose how to replace center $n_{k, 1}$. Return this new cell neighborhood $\mathbf{n}$.

Input: $\tau$ : Tournament size, $\mathbf{X}$ : Input training dataset, $\beta$ : Mutation probability, $\mathbf{n}$ : Cell neighborhood sub-population, $M$ : Loss functions

Return: $\mathbf{n}$ : Cell neighborhood sub-population

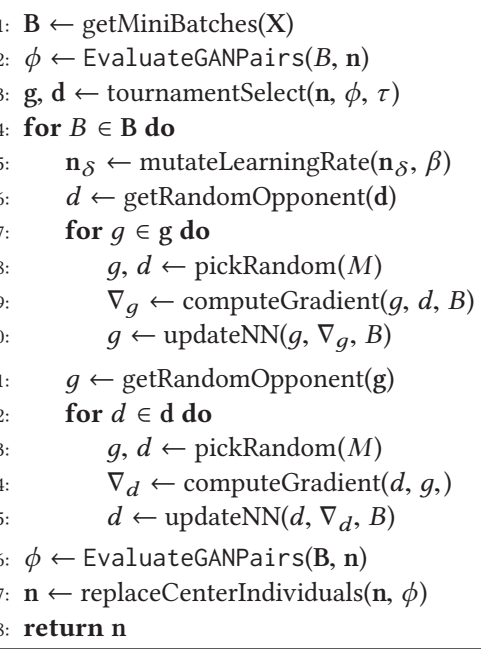

$\triangleright$ Load minibatches

$\triangleright$ Evaluate all updated GAN pairs, Alg. 4 $\triangleright$ Select using $\operatorname{loss}(\mathcal{L})$ as fitness $\triangleright$ Loop over batches

$\triangleright$ Update neighborhood learning rate with with Gaussian mutation

$\triangleright$ Get uniform random discriminator

$\triangleright$ Evaluate generators and train with SGD

$\triangleright$ Random draw of loss function for each generator and discriminator

$\triangleright$ Compute gradient for neighborhood center $\triangleright$ Update with gradient

$\triangleright$ Get uniform random generator

$\triangleright$ Evaluate discriminator and train with SGD

$\triangleright$ Random draw of loss function for each generator and discriminator

$\checkmark$ Compute gradient for neighborhood center $\triangleright$ Update with gradient

$\triangleright$ Evaluate all updated GAN pairs, Alg. 4 $\triangleright$ Best generator and discriminator are placed in the center based on loss

ALGORITHM 4: EvaluateGANPairs: Evaluate all models against each other using the "best worst case" solution concept $f_{s}^{\text {min }}$ based on the training loss $\mathcal{L}$

Input: $\mathbf{B}$ : Minibatches, $\mathbf{n}$ : Cell neighborhood sub-population

Return: $\phi$ : Generator and discriminator fitnesses, based on training loss $\mathcal{L}$

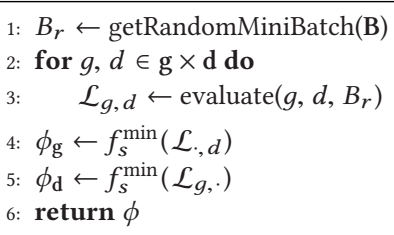

$\triangleright$ Get random minibatch

$\triangleright$ Evaluate all GAN pairs $\triangleright$ Evaluate GAN

$\triangleright$ Fitness for generator is the "best worst case" loss value $(\mathcal{L})$ $\triangleright$ Fitness for discriminator is the "best worst case"loss value $(\mathcal{L})$

GAN training. In Lipizzaner GAN training is also a black-box component because, as long as model parameters are updated and a learning rate $\delta$ can be specified parametrically, the coevolutionary algorithm is oblivious to how they are updated or used, Algorithm 3::CoevolveAndTrainModels lines 9, 10, 14, and 15. Therefore, we set up the three loss functions described in Section 3 with corresponding optimization objectives and randomly assign one to a model after selection.

\subsection{Discussion of Algorithmic Design and Implementation}

Lipizzaner is open source with an MIT license. ${ }^{1}$ It is written in Python and uses PyTorch. The source is available from https://github.com/ALFA-group/lipizzaner-gan. Note that depending on

\footnotetext{
${ }^{1}$ https://mit-license.org/.
} 
how long a generation takes to compute, the asynchronicity can have varying impact on the performance of Lipizzaner.

Evolutionary algorithms (EAs) are black-box algorithms capable of solving problems viewed in terms of their inputs and outputs, without any knowledge of internal workings. In Lipizzaner, GAN evaluation is a black-box component, because the algorithm can query an opaque module with some inputs and get back outputs that describe performance. We make this point to emphasize the generality of the hybridization between GANs and a competitive evolutionary algorithm. Any GAN model evaluation component could work within Lipizzaner. We implement particular GAN examples, without loss of generality.

In this version of Lipizzaner, we define the black box to deliberately randomize and vary the loss function of the training at the cell level. This will, in part, address the dilemma of not knowing what function is ideal and being forced into only choosing one. Another effect of multiple loss functions could be that they promote diversity in the trained models samples. The belief is that when all models always use the same loss function, there is a risk that Lipizzaner will converge prematurely.

Evaluation. Note that the generator ensemble e selection fitness, FID score $q$, is intentionally different from the GAN training fitness $\phi$ measured on the discriminator $d$ and generator $g$ during GAN training. This is because, during training, the loss must reflect the adversarial paradigm where the generator is guided to "fool" the discriminator. Once the adversarial training is over, the generator must be evaluated on its ability to capture the distribution $\mathcal{T}^{*}$ of the data, which is measured by its ability to generate images that look similar to the sample images. See Heusel et al. [2017b], which serves as a precedent. This reasoning also explains why there is no training and test split of the dataset, i.e., training is guided to discriminate real from fake using all the samples, while testing inputs are from the latent space and the evaluation criteria, FID, is similarity to the samples.

Communication. The only active communication in Lipizzaner is each cell's read of its four adjacent cells to form the model sub-populations at the start of each generation. There is no explicit inter-cell synchronization. This makes the software convenient to deploy on heterogenous hardware and training that may exhibit varying execution times. Passive communication occurs when each cell updates itself, thus allowing the cells that consider it to be their neighbor to access its new state.

Complexity Analysis. Using the notation in Table 1, the computational cost of Lipizzaner is the product of costs at the cell level and the number of cells in the grid, $N$, and the number of generations $T$. Cell-level cost can be decomposed into fitness evaluations before and after training, GAN training itself per generation, and measurement score calculation when evaluating the performance of the mixture weights. Other computation at the cell is insignificant. The cost of fitness evaluations before Algorithm 3 and after training is twice the product of evaluating the loss of every model against its adversary. This entails $s^{2}$ loss calculations. Batch training iterates to use the entire dataset, $\mathrm{X}$ with size $e$, once. Each iteration there are $2 s$ GAN trainings. Each GAN training $\operatorname{cost} C$ entails back propagation to obtain gradient information and network parameter updates.

The computational complexity of cell-level effort is dominated by GAN training. Therefore, the computational complexity of Lipizzaner is $O\left(s^{2} N T C e\right)$. If we consider the von Neumann neighborhood size $s$ a constant, $s=5$, then the complexity simplifies to $O(N T C e)$.

\section{EMPIRICAL EXPERIMENTS AND RESULTS}

We now address the two research questions stated in Section 1. Section 5.1 describes our general experimental configurations. Section 5.2 details the ablation experiments to address RQ-1: Are 
Table 2. Setup for Experiments Conducted with the Lipizzaner System on MNIST and CelebA Datasets

\begin{tabular}{|c|c|c|}
\hline Parameter & MNIST & CelebA \\
\hline \multicolumn{3}{|c|}{ Coevolutionary settings } \\
\hline Generations (epochs), $T$ & 200 & 20 \\
\hline Tournament size, $\tau$ & 2 & 2 \\
\hline Grid size & \multicolumn{2}{|c|}{ See Table 6} \\
\hline Mixture weight mutation step & 0.01 & 0.05 \\
\hline \multicolumn{3}{|c|}{ Parameter mutation } \\
\hline Initial learning rate, $\delta$ & 0.0002 & 0.00005 \\
\hline Mutation step & 0.0001 & 0.0001 \\
\hline Mutation probability, $\beta$ & 0.5 & 0.5 \\
\hline \multicolumn{3}{|c|}{ Network topology (for generator and discriminator) } \\
\hline Network type & MLP & DCGAN \\
\hline Input neurons & 64 & 100 \\
\hline Number of hidden layers & 2 & 4 \\
\hline Neurons per hidden layer & 256 & $16,384-131,072$ \\
\hline Output neurons & 784 & $64 \times 64 \times 3$ \\
\hline Activation function & $\tanh$ & $\tanh$ \\
\hline \multicolumn{3}{|c|}{ Training settings } \\
\hline Optimizer & Adam & Adam \\
\hline Batch size & 100 & 128 \\
\hline
\end{tabular}

each of the different features of Lipizzaner's evolutionary computation methodology are needed? Section 5.3 presents experiments with different loss functions to address RQ-2: Do different loss function combinations result in models with better performance and higher diversity of solutions and model parameters?

\subsection{Experimental Configurations}

Experiment GAN and coevolutionary algorithm parameter settings are shown in Table 2. E-GAN and GAN-BCE both use the Adam optimizer with an initial learning rate (0.0002). We follow Wang et al. [2019] to set up the remaining parameters of E-GAN. All methods have been implemented in Python 3 and pytorch. ${ }^{2}$ The experiments are performed on a cloud that provides eight Intel Xeon cores, $2.2 \mathrm{GHz}$, with 32 GB RAM and an NVIDIA Tesla T4 GPU with 16 GB RAM. All implementations use the same Python libraries and versions to minimize computational differences that could arise from using the cloud. We consider the variances that could arise to be insignificant.

We use two common image datasets from the GAN literature: MNIST [LeCun 1998] and CelebA. MNIST has been widely used and is especially appropriate to investigate mode collapse due to its limited target space (namely, the characters 0-9). The larger CelebA dataset [Liu et al. 2015] contains 200,000 images of faces. To obtain an absolute measure of model performance, we draw fake image samples from the generators and score them with FID [Heusel et al. 2017b]. FID score is a black box, discriminator-independent, non-linear measure that expresses image similarity to the samples used in training. Note, lower FID score is better, hence, we perform minimization.

For Lipizzaner, we also investigate the diversity of the evolved networks to see whether one network is replicated over the entire grid. One measure of the spatial topology property is the max

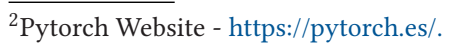


Table 3. Lipizzaner Feature Ablation

\begin{tabular}{l|c|c|c|c}
\hline Ablation & Communication & Selection & Ensemble & Learning Rate Mutation \\
\hline Baseline & $\checkmark$ & $\checkmark$ & $\checkmark$ & $\checkmark$ \\
No Learning Rate Mutation & $\checkmark$ & $\checkmark$ & $\checkmark$ & $\checkmark$ \\
No Ensemble Weight Optimization & $\checkmark$ & $\checkmark$ & & $\checkmark$ \\
No Local Selection & $\checkmark$ & & $\checkmark$ & $\checkmark$ \\
No Selection and Communication & & & $\checkmark$ & $\checkmark$ \\
All But Ensemble Weight Optimization & & & $\checkmark$ & \\
\hline
\end{tabular}

Columns indicate $\checkmark$ if the ablation is using the feature. Rows indicate the different ablations.

neighborhood distance $\left(\Delta_{n}^{\max }\right)$. Grids smaller than $4 \times 4(m<4)$ always have neighborhood overlap, i.e., the $\Delta_{n}^{\max }$ is 0 . For $4 \times 4$ the $\Delta_{n}^{\max }$ is $1,(4-3)$, and for $5 \times 5$ the $\Delta_{n}^{\max }$ is $2(5-3)$.

\subsection{Ablation Experiments}

The goal of RQ-1 and experiments in this section is to establish the relevance of Lipizzaner's features. We proceed by means of ablation. In Section 5.2.1, we outline our ablation setups and in Section 5.2.2, we show and discuss the results.

5.2.1 Ablation Setup. We conduct the following ablation experiments, all using BCE as the loss function for both the generator and discriminator, and 30 independent runs. The baseline is Lipizzaner with all features present. The ablations are listed in Table 3. They consist of single ablations for each of selection, ensemble weight optimization, and learning rate mutation. Eliminating communication only is meaningless; therefore, we ablate both selection and communication together. Finally, we ablate all but ensemble weight optimization to gauge its standalone merit. Note, we always use SGD when training the GANs.

The ablations (see Table 3) are:

- No Learning Rate Mutation: Lipizzaner without evolutionary mutation of the initial value of the Adam optimizer learning rate (0.0002) (see Algorithm 2::MixtureEA).

- No Local Selection: Lipizzaner without selection at the local neighborhood scale (see line 3 of Algorithm 3::CoevolveAndTrainModels).

- No Ensemble Weight Optimization: Lipizzaner without ensemble weight optimization though still with an ensemble solution. The generative model returned is defined by the five generators in the most competitive neighborhood with the same mixture weights (i.e., $\omega_{i}=0.2$ ) (see line 7 of Algorithm 1::Lipizzaner).

- No Selection and Communication: Lipizzaner without selection and communication but using learning rate mutation and ensemble weight optimization. We run Lipizzaner $1 \times 1$ (i.e., $1 \times 1-G A N-B C E) 30$ times with learning rate mutation. Then, we randomly select subsets of five generators to create mixtures by applying ensemble weight optimization.

- All But Ensemble Weight Optimization: Lipizzaner without selection and communication and learning rate mutation, but using ensemble weight optimization. Our goal is to approximate Lipizzaner executing with random search, i.e., only GAN training. Thus, we train 30 GANs (GAN-BCE) individually. Then, we randomly select subsets of five generators to create mixtures by applying ensemble weight optimization.

We choose the $4 \times 4$ grid size to have minimal overlap of the neighborhoods to isolate the effects of communication component in Lipizzaner. We measure the effect of the different variants using FID score. 
Table 4. FID Results for Lipizzaner Ablation Experiments on MNIST-4 $\times 4$ Given Fixed Number(200) of Generations

\begin{tabular}{|c|c|c|c|c|c|c|}
\hline Ablation & Mean & Std & edian & $\Delta \mathrm{IQR}$ & Min & Max \\
\hline Baseline: all features present & 29.11 & 5.20 & 29.41 & 6.51 & 19.56 & 40.42 \\
\hline No Learning Rate Mutation & 45.64 & 13.65 & 41.43 & 9.88 & 30.75 & 99.23 \\
\hline No Local Selection & 37.97 & 8.89 & 35.98 & 13.61 & 23.69 & 56.36 \\
\hline No Ensemble Weight Optimization & 36.57 & 5.41 & 35.90 & 3.91 & 26.35 & 54.60 \\
\hline No Selection and Communication & 35.94 & 2.72 & 36.30 & 3.80 & 28.03 & 39.32 \\
\hline All But Ensemble Weight Optimization & 49.44 & 4.08 & 43.13 & 6.27 & 49.16 & 58.50 \\
\hline
\end{tabular}

$\triangle \mathrm{IQR}$ is the inter-quartile range difference, the difference between the 75 th percentile and the 25 th percentile. The baseline, where all features are present, has the statistically significant lowest (best) FID score, minimum, median and mean. Lowest value is in bold.

5.2.2 Ablation Results. The results from the ablation experiments on MNIST $-4 \times 4$ are shown in Table 4. For any single ablation the experimental average mean, median, minimum, and maximum FID score are worse than the baseline. Moreover, the baseline is statistically better than all the other methods according to pairwise Wilcoxon Rank Sum statistical test $(\alpha<0.01)$.

As would be expected, the impact of ablating mutation of the initial value of the Adam learning rate is the most severe. Both GAN and network model training convergence and performance are extremely sensitive to initial conditions. When both selection and communication are eliminated, while learning rate mutation and ensemble weight optimization remain, we obtain an average mean FID score that is closer to the baseline than any single ablation. Finally, when we retain only ensemble weight optimization (bottom row of Table 4), we can see the effect of the learning rate mutation in providing better results. These findings verify the importance of each of the components in Lipizzaner for its performance. Note that recent work Toutouh et al. [2020] shows that weight optimization of the ensemble done only at the end provides similar results to the Baseline. In the public release of Lipizzaner the choice to evolve weights during training is now parameterized.

\subsection{Using Different Loss Functions}

RQ-2 asks whether different loss function combinations result in models with better performance and more diversity. This provides guidance as to whether developers, lacking knowledge of the ideal loss function to train with, should try different Lipizzaner combinations. In Section 5.3.1, we set up pairwise combinations of generator and discriminator loss functions that are used at every cell throughout the evolutionary run. We also experiment with randomizing the combination used in each generation by any cell. In Section 5.3.2, we compare the results, and in Section 5.3.3, we check for diversity in the trained model parameters and in the distribution estimated by the best trained ensemble.

5.3.1 Experimental Setup. We configure different GAN training setups. The baselines are not spatially distributed (WGAN, GAN-BCE, and E-GAN). WGAN and GAN-BCE are also the only combinations without a population (see Cheng et al. [2020] for a comprehensive analysis of GANs used on MNIST). The Lipizzaner variants use either the same loss functions (see Section 3) for generatordiscriminator for all training cycles (BCE-BCE, MSE-BCE, MSE-MSE, HEU-HEU, WASS-WASS) or not (RAND-COMBOS).

The setups are described in Table 5. Note that the combinations extend Toutouh et al. [2019], which did not randomize the loss function of discriminator and instead always used BCE for the discriminator. E-GAN [Wang et al. 2019], which we describe in Section 2, is also included. 
Table 5. Variants for Comparing GAN Training Performance

\begin{tabular}{|c|c|c|c|c|}
\hline Name & \begin{tabular}{|c|} 
Spatial \\
Distribution
\end{tabular} & \begin{tabular}{|c|}
$\begin{array}{c}\text { Loss Function } \\
\text { Diversity }\end{array}$ \\
\end{tabular} & Generator & Discriminator \\
\hline \multicolumn{5}{|c|}{ Baselines } \\
\hline GAN-BCE & & & $\mathrm{BCE}$ & BCE \\
\hline WGAN [Arjovsky et al. 2017] & & & WASS & WASS \\
\hline E-GAN [Wang et al. 2019] & & $\checkmark$ & BCE, MSE, HEU & BCE \\
\hline \multicolumn{5}{|c|}{ Loss Function Combinations } \\
\hline BCE-BCE & $\checkmark$ & & $\mathrm{BCE}$ & $\mathrm{BCE}$ \\
\hline MSE-BCE & $\checkmark$ & & MSE & $\mathrm{BCE}$ \\
\hline MSE-MSE & $\checkmark$ & & MSE & MSE \\
\hline HEU-HEU & $\checkmark$ & & $\mathrm{HEU}$ & $\mathrm{BCE}$ \\
\hline WASS-WASS & $\checkmark$ & & WASS & WASS \\
\hline RAND-COMBOS & $\checkmark$ & $\checkmark$ & BCE, MSE, HEU & BCE, MSE \\
\hline
\end{tabular}

Ability to train with distribution and different loss function combinations is indicated with $\checkmark$. The loss functions used for generator and discriminator are also listed, see Section 3.

Table 6. Lipizzaner Grid Sizes Used for the Experiments on Different Datasets

\begin{tabular}{l|ccc|c}
\hline & \multicolumn{3}{|c}{ MNIST } & CelebA \\
Name & $3 \times 3$ & $4 \times 4$ & $5 \times 5$ & $2 \times 2$ \\
\hline BCE-BCE & $\checkmark$ & $\checkmark$ & $\checkmark$ & $\checkmark$ \\
MSE-BCE & $\checkmark$ & & & \\
MSE-MSE & $\checkmark$ & $\checkmark$ & & $\checkmark$ \\
HEU-HEU & $\checkmark$ & $\checkmark$ & & $\checkmark$ \\
WASS-WASS & & $\checkmark$ & & \\
\hline RAND-COMBOS & $\checkmark$ & $\checkmark$ & $\checkmark$ & $\checkmark$ \\
\hline
\end{tabular}

Sizes were selected based on a facet-wise approach. The name indicates which loss functions (Section 3) were used for generator and discriminator.

To compare the impact of different loss functions, we execute all methods with a computational budget of nine hours. The spatial coevolutionary methods of RAND-COMBOS, BCE-BCE, MSE-MSE, and HEU-HEU use a grid size of $3 \times 3$ and are able to train nine networks in parallel. Thus, they are simultaneously executed for one hour.

In addition, we also further investigated different grid sizes. Table 6 shows what size grids were investigated for each of the different methods. Computational costs do not allow a full grid comparison, so we isolate specific combinations and sizes.

We quantify the distributional coverage of the trained ensemble's fake images in two ways. Per Li et al. [2017a], we report their Total Variation Distance (TVD):

$$
T V D=\frac{1}{2} \sum_{l \in L}\left|\left\|X_{l}\right\|-\left\|d(z)_{l}\right\|\right|,
$$

where $L$ are the labels (classes), $\left\|X_{l}\right\|$ the frequency (number) of labels in the data $(X)$, and $\left\|d(z)_{l}\right\|$ the frequency (number) of labels in the generated data $D_{d}\left(G_{g}(z)\right)$. The labels of the generated data are determined using a pre-trained classifier.

We also visually inspect the appearances of fake images for each class of the dataset, noting that this method depends on a dataset with classes. Finally, to assess whether the different combinations result in different networks, we calculate the $L_{2}$ distance between network parameters. 
To gauge the benefits of the different variants, if any, we execute 30 independent runs of each method in Table 5 on the MNIST problem with equivalent computational budgets. At the end of each run, we report the performance of the best performing mixture model (see Table 7) as FID score where lower is better.

5.3.2 Loss Function Combination Results. Preliminarily, the benefit of a population over a single GAN is discernible. The FID scores of GAN-BCE, the single GAN trained for 9 hours, and E-GAN, where a single GAN is replicated and trained with three loss functions before selection, are surpassed by all of Lipizzaner's population-based combinations. GAN-BCE's mean FID score is approximately 10 times higher (worse) and its lowest/best FID score is significantly higher (worse), approximately 10 times. These differences are statistically significant ( $p$-value $<0.01$ ) according to a Wilcoxon ranksum test. All of the Lipizzaner methods perform statistically better than E-GAN, GAN-BCE, and WGAN. Focusing on GAN-BCE and BCE-BCE, which train with the same loss function pair, the advantage of distributed spatial evolution is clearly apparent. They have comparable FID scores during the first 30 training epochs, however, BCE-BCE improves in performance over the remaining epochs (not shown).

We note that the mean and median FID scores of GAN-BCE are better than E-GAN, and E-GAN's performance demonstrates a larger variance of FID scores compared to GAN-BCE. It should, however, be noted that, in the original paper, Wang et al. [2019], which used a computational budget of $30 \mathrm{~h}$ compared to the $9 \mathrm{~h}$ we use here, E-GAN performance keeps improving. The results provided by Lipizzaner variants that generate diversity only by a spatial population (BCE-BCE, HEU-HEU, and MSE-MSE) are significantly more competitive than E-GAN, which experiments with loss function diversity but does not evolve a population from one generation to the next. Note that the performance of E-GAN compared to Lipizzaner provided basis for our decision to focus our computational resources on investigating Lipizzaner.

The experiments of Table 7, along with Table 8, which show FID scores for different grid sizes, allow us next to answer whether explicitly promoting diversity with RAND-COMBOS makes it superior among the other Lipizzaner variants in terms of performance. Recall that they use different, static, pairwise combinations of loss functions for gradient-based GAN training. While we did not exhaustively try every combination of static pairs, it is arguably possible that one static pairing will surpass RAND-COMBOS, because randomization introduces noise. We do not observe this in most measures in Table 7. An exception is RAND-COMBOS's $\triangle I Q R$, which is outscored by BCE-BCE. A Wilcoxon ranksum test with Holm correction confirms that the difference between RAND-COMBOS and the other methods for MNIST-3 $\times 3$ is significant at confidence levels between $\alpha=0.001$ and $\alpha=0.05$. Across three grid sizes, per Table 8, rank ordering changes among the methods, however no statistically superior method emerges. However, because RAND-COMBOS both explicitly promotes diversity by randomly assigning loss functions dynamically, i.e., before each training batch, and it avoids the necessity of exhaustive, static pairwise investigation, it is arguably a better choice.

A secondary question is how efficiently each method uses the same computational budget of $9 \mathrm{~h}$. Lipizzaner variants, including RAND-COMBOS, execute asynchronously and in parallel for all the cells. There is no bottleneck between cells. E-GAN has a synchronization bottleneck, because the three copies of the generator are trained sequentially with a single copy of the discriminator. Because Lipizzaner variants execute with asynchronous parallelism, the number of training epochs performed by each cell of the grid in the same run varies. The number of epochs are normally distributed for all the algorithms (not shown). As expected GAN-BCE, a serially trained, single GAN trains for the most epochs. It performs about two times the number of generations of E-GAN, which evaluates three generator networks each generation. The spatially distributed coevolutionary algorithms performed significantly fewer training epochs than E-GAN. However, during each epoch 
Table 7. FID Values for 30 Independent Runs of MNIST-3 $\times 39$ h Compute Time for Different GAN Training Methods

\begin{tabular}{lrrrrrr}
\hline & Mean & \multicolumn{1}{c}{ Std } & Median & \multicolumn{1}{c}{$\Delta$ IQR } & \multicolumn{1}{c}{ Min } & \multicolumn{1}{c}{ Max } \\
\hline E-GAN [Wang et al. 2019] & 466.11 & 48.89 & 481.61 & 69.33 & 277.47 & 504.86 \\
GAN-BCE [Goodfellow 2016] & 448.31 & 28.69 & 453.53 & 21.69 & 326.11 & 474.56 \\
WGAN [Arjovsky et al. 2017] & 180.89 & 104.97 & 161.48 & 13.22 & 146.05 & 733.87 \\
BCE-BCE & 48.96 & 10.00 & 46.07 & $\mathbf{4 . 6 6}$ & 38.01 & 80.88 \\
MSE-MSE & 52.30 & 8.89 & 54.13 & 14.98 & 37.51 & 65.09 \\
HEU-HEU & 52.52 & 9.20 & 52.73 & 9.77 & 34.87 & 72.43 \\
RAND-COMBOS & $\mathbf{4 2 . 2 4}$ & $\mathbf{5 . 5 3}$ & $\mathbf{4 3 . 1 8}$ & 7.59 & $\mathbf{3 0 . 7 7}$ & $\mathbf{4 8 . 9 8}$ \\
\hline
\end{tabular}

RAND-COMBOS has the significantly best performance. ( $\triangle \mathrm{IQR}$ is the inter-quartile range difference). Lowest value is in bold.

Table 8. FID Results for MNIST Experiments with Different Grid Sizes for Lipizzaner Variants Given Fixed Number (200) of Generations

\begin{tabular}{|c|c|c|c|c|c|c|}
\hline & Mean & Std & Median & $\triangle \mathrm{IQR}$ & Min & Max \\
\hline \multicolumn{7}{|c|}{ MNIST-3 × 3 (Population 9) } \\
\hline RAND-COMBOS & 39.51 & 5.24 & 39.48 & 8.54 & 25.40 & 48.13 \\
\hline BCE-BCE & 40.78 & 7.74 & 40.27 & 12.59 & 26.68 & 58.76 \\
\hline MSE-MSE & 49.79 & 8.65 & 50.76 & 8.12 & 35.59 & 66.96 \\
\hline HEU-HEU & 43.26 & 7.22 & 42.33 & 5.05 & 30.88 & 60.40 \\
\hline \multicolumn{7}{|c|}{ MNIST-4 × 4 (Population 16) } \\
\hline RAND-COMBOS & 30.29 & 3.71 & 30.46 & 4.13 & 21.23 & 38.29 \\
\hline BCE-BCE & 29.11 & 5.20 & 29.41 & 6.51 & 19.56 & 40.42 \\
\hline MSE-MSE & 29.14 & 4.32 & 28.98 & 6.36 & 22.31 & 37.45 \\
\hline HEU-HEU & 30.28 & 8.32 & 31.14 & 13.04 & 18.57 & 55.22 \\
\hline WASS-WASS & 148.36 & 7.92 & 150.47 & 9.97 & 127.86 & 161.47 \\
\hline \multicolumn{7}{|c|}{ MNIST-5 × 5 (Population 25) } \\
\hline RAND-COMBOS & 26.78 & 2.47 & 27.65 & 2.03 & 21.85 & 29.46 \\
\hline BCE-BCE & 28.16 & 4.05 & 28.13 & 5.81 & 22.56 & 40.01 \\
\hline
\end{tabular}

for $3 \times 3$, these methods evaluate 45 GANs, i.e., neighborhood size of 5 on 9 cells, which is 15 times more generator and discriminator networks than E-GAN.

The best FID for the different grid sizes and loss functions are shown in Table 8. As expected, the larger grid sizes impact the FID more than the use of loss functions. RAND-COMBOS has consistently a good performance and low variance for the different grid sizes, i.e., it makes good use of the increase in population size (and implicitly the number of fitness evaluations) and max neighbor distance, $\Delta_{n}^{\max }$. Note that we only use the two best Lipizzaner versions for $5 \times 5$.

From these results, we observe: (A) WGAN improves GAN-BCE. (B) WASS-WASS improves WGAN. (C) WASS-WASS does not improve Lipizzaner. This can be due to observations from the literature [Lesort et al. 2019] that reported that MNIST WGAN is less stable than GAN-BCE, as can be seen by the Std in Table 7. Note, WASS-WASS is not included in the FID box-plot to avoid losing information due to the differences in scale.

The median FID over each training epoch is shown in Figure 4. The FID values are smoother the larger the population size. Note that the total number of fitness evaluations (FE) are different 


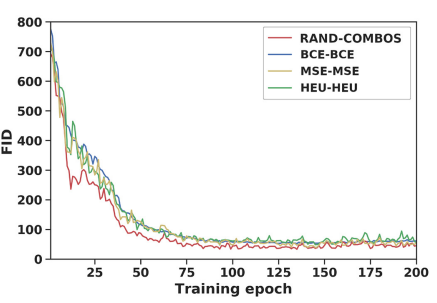

(a) MNIST-3 $\times 3$ (population 9)

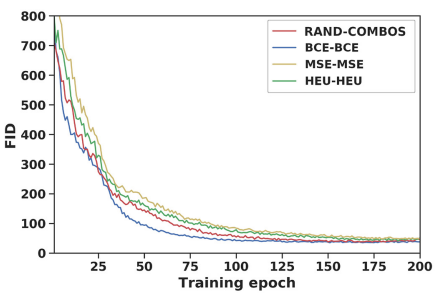

(b) MNIST-4×4 (population 16)

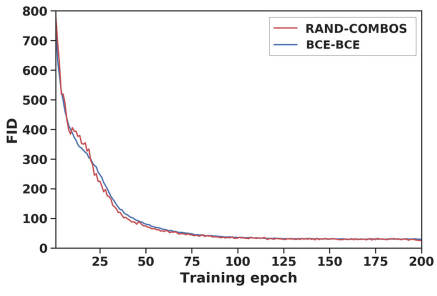

(c) MNIST-5×5 (population 25)

Fig. 4. Results on MNIST dataset for different grid sizes. Median FID (y-axis) evolution through the epochs (X-axis), each line is a different Lipizzaner variant.

Table 9. FID Results on CelebA Dataset on a $2 \times 2$ Showing Best FID Mean, Normalized Standard Deviation, Median, and Inter-quartile Range Difference $(\triangle \mathrm{IQR})$

\begin{tabular}{lrrrrrr}
\hline & Mean & \multicolumn{1}{c}{ Std } & Median & \multicolumn{1}{c}{$\Delta$ IQR } & Min & \multicolumn{1}{c}{ Max } \\
\hline RAND-COMBOS & $\mathbf{3 6 . 1 7}$ & $\mathbf{0 . 7 8}$ & 35.83 & $\mathbf{0 . 9 7}$ & 35.35 & $\mathbf{3 7 . 2 9}$ \\
BCE-BCE & 36.18 & 1.93 & $\mathbf{3 5 . 3 9}$ & 1.64 & 34.64 & 39.75 \\
MSE-BCE & 163.11 & 118.34 & 123.13 & 191.54 & 55.41 & 310.79 \\
HEU-HEU & 38.35 & 2.76 & 39.17 & 4.50 & 34.94 & 40.93 \\
GAN-BCE & 137.78 & 143.00 & 44.54 & 214.20 & $\mathbf{2 9 . 3 1}$ & 365.43 \\
\hline
\end{tabular}

Low FID indicates good performance and lowest value is in bold.

for each grid size $4 \times 4$ has $\approx 1.8$ more FEs than $3 \times 3$, and $5 \times 5$ has $\approx 2.2$ more FEs than $3 \times 3$. When looking at the FIDs for the different grid sizes, we see that the median values improvement is correlated to the increase in number of fitness evaluations.

Finally, we compare the spatially distributed coevolutionary Lipizzaner methods as applied to generate the CelebA dataset. We chose these methods, since they had good performance on the MNIST dataset. Table 9 summarizes the results over six independent runs. The last row shows the results of training a single GAN-BCE using the same computational resources as the distributed methods, i.e., it performs four times the number of training epochs of the distributed methods. RAND-COMBOS provides the lowest median FID and MSE-MSE the highest one. BCE-BCE and HEU-HEU provide median and average FID scores close to the RAND-COMBOS ones. However, RAND-COMBOS is more robust to the varying performance of the methods that apply a unique loss function (see Std and $\triangle I Q R$ in Table 9). Even though GAN-BCE finds the generative model with the lowest FID, its variance indicates that most of the time it suffers from GAN training pathologies. Note that Lipizzaner does not achieve state-of-the-art CelebA performance with these experimental settings and GAN architecture; the point is to show the impact of Lipizzaner variants. In Figure 5, we show how BCE-BCE can recover from generator collapse with sampled images for the training iteration before, during, and after a generator collapse.

In summary, generally these results indicate that spatially distributed coevolutionary training is the best choice to train GANs, even when there is no knowledge about the best loss function to the problem. RAND-COMBOS, the best method for promoting diversity, is best at utilizing the increase in fitness evaluations through the increase of grid size to improve the FID.

5.3.3 Diversity through Different Loss Function Combinations. We start by examining the extent of diversity in different methods. We contrast the Lipizzaner variants that generate diversity by a spatial population (BCE-BCE, HEU-HEU, and MSE-MSE) to E-GAN, which explicitly promotes 


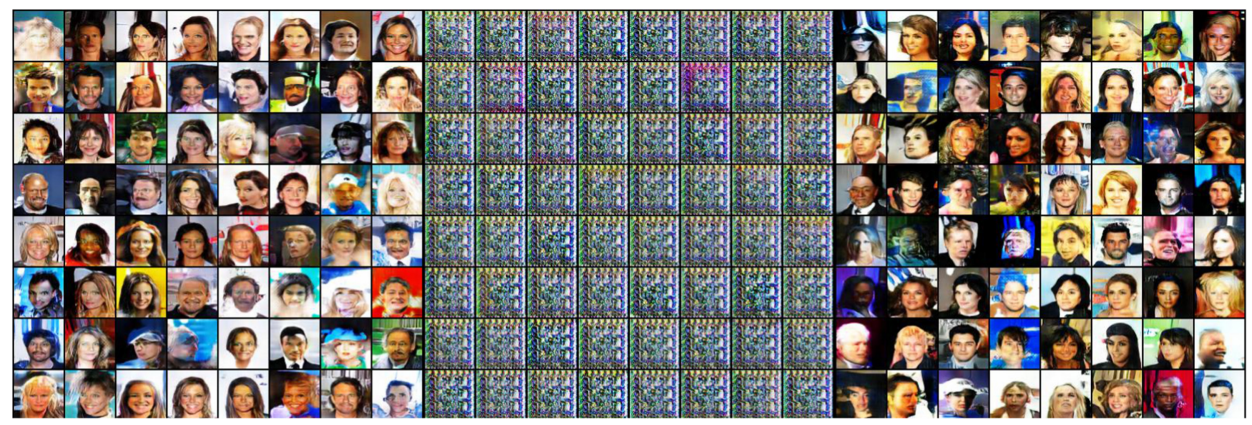

(a) CelebA $2 \times 2$ before collapse

(b) CelebA $2 \times 2$ collapse

(c) CelebA $2 \times 2$ after collapse

Fig. 5. Generated images for CelebA for BCE-BCE iterations before, during, and after a collapse.

Table 10. TVD Measurements on MNIST Dataset

\begin{tabular}{lccccc}
\hline Experiment & RAND-COMBOS & BCE-BCE & HEU-HEU & MSE-MSE & WASS-WASS \\
\hline$M N I S T-3 \times 3$ & $\mathbf{0 . 1 2 2}(0.016)$ & $0.138(0.028)$ & $0.144(0.034)$ & $0.145(0.032)$ & NA \\
$M N I S T-4 \times 4$ & $\mathbf{0 . 1 2 1}(0.021)$ & $0.124(0.028)$ & $0.128(0.031)$ & $0.131(0.032)$ & $0.142(0.018)$ \\
$M N I S T-5 \times 5$ & $\mathbf{0 . 1 0 8}(0.022)$ & $0.118(0.020)$ & NA & NA & NA \\
\hline
\end{tabular}

Each row shows mean (std) TVD values, low TVD indicates more diversity, for different grid sizes. The columns show the Lipizzaner variants. RAND-COMBOS consistently has the lowest TVD value. Lowest value is in bold.

additional diversity by loss function randomization each epoch. We use two measures of output diversity, neither better than the other, simply different in what characteristic of diversity they tally: TVD (lower is better) and class balance. For MNIST, ideally each digit class is represented with the same frequency in the fake image samples. Additionally, we use a measure of model diversity where we look at network parameters from one cell to the others.

In considering diversity in a set of generator-derived output samples (images in this case), our hypothesis is that the RAND-COMBOS will exhibit higher output diversity than the other Lipizzaner methods, because the randomization of the training loss function impacts the convergence rate of training. Figure 6 shows a random selection of generator sample fake images for each method. Each Lipizzaner method, a spatially distributed coevolutionary algorithm, is able to produce a mixture that generates what visually appears to be well-balanced and accurate-looking digits.

The total variation distance (TVD) for each algorithm [Li et al. 2017a] is shown in Table 10 and Figure 7. Decrease in TVD values, i.e., diversity, increases with the grid size for each method. Moreover, RAND-COMBOS consistently has the lowest TVD value, i.e., diversity of the generative model at every grid size vs. the other Lipizzaner methods that do not promote diversity through loss function randomization.

The distributions of each digit class for generated samples show that HEU-HEU and RAND-COMBOS generate samples spanning across different classes. The two methods (E-GAN, GAN-BCE) that do not promote diversity via spatial distribution suffer from what is possibly mode collapse. About half of the samples are the digit 3, and they do not generate samples of digits 4 and 7 .

We next measure the output diversity of generators trained with MNIST by measuring the class distributions. Figure 8 shows the absolute value of the difference between the proportion of the generated samples and the ideal proportion (10\%) for each class. The difference in original and generated class distribution decreases when the size of the grid increases, implying that spatial distribution contributes to diversity. RAND-COMBOS, which explicitly adds an additional means of 


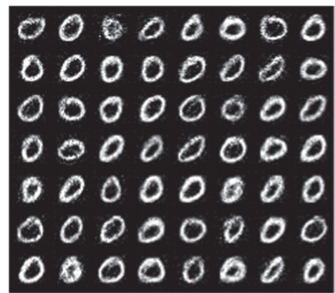

(a) Mode collapse

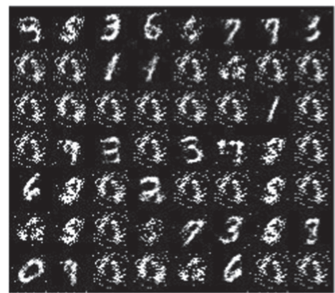

(e) MSE-BCE

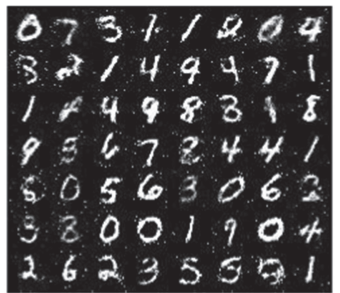

(b) RAND-COMBOS

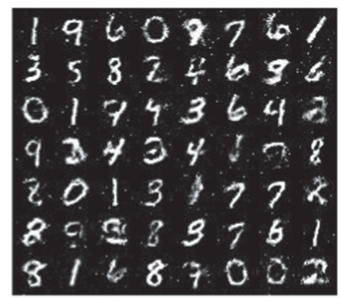

(c) BCE-BCE

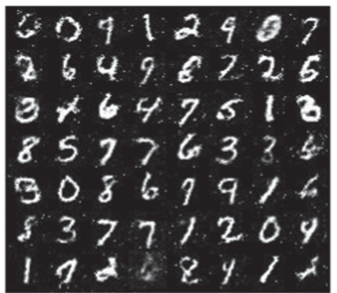

(d) HEU-HEU

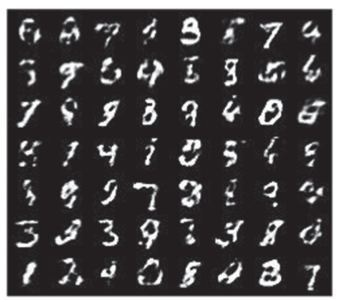

(f) WASS-WASS

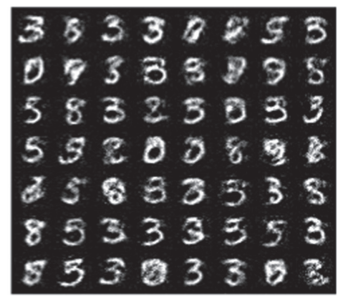

(g) E-GAN

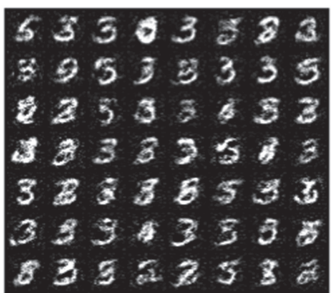

(h) GAN-BCE

Fig. 6. Sequence of samples generated of MNIST dataset. (a) mode collapse, the generator is focused on the character 0 . It illustrates samples generated by the best generator (in terms of FID, see Table 8 for values) by RAND-COMBOS (b), BCE-BCE (c), HEU-HEU (d), MSE-MSE (e), WASS-WASS (f), E-GAN (g), and GAN-BCE (h).

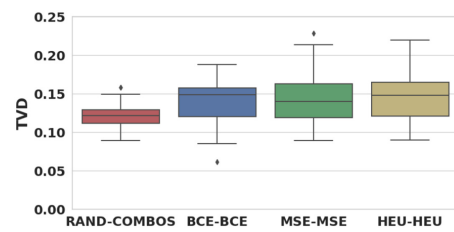

(a) $M N I S T-3 \times 3$

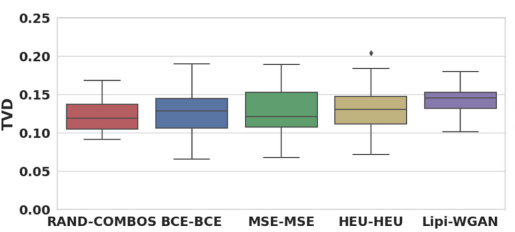

(b) $M N I S T-4 \times 4$

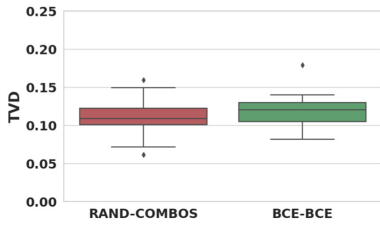

(c) MNIST $-5 \times 5$

Fig. 7. Box-plots of TVD results on MNIST dataset for different grid sizes (Low TVD indicates more diversity). TVD is on Y-axis and Lipizzaner variant on Y-axis.

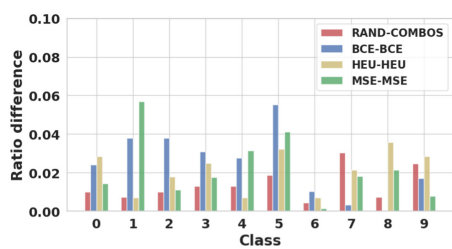

(a) $M N I S T-3 \times 3$

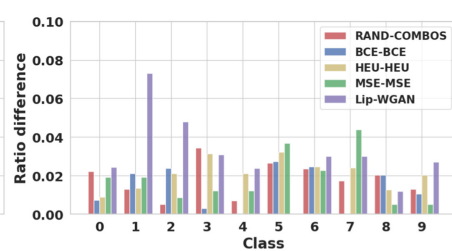

(b) $M N I S T-4 \times 4$

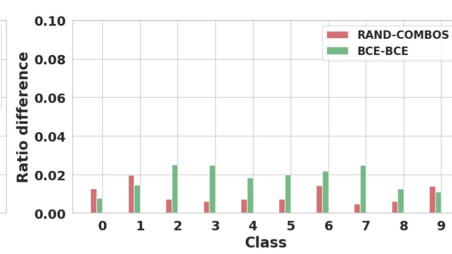

(c) $M N I S T-5 \times 5$

Fig. 8. Absolute per class value (x-axis) of the difference between the ratio of the generated distribution and the ratio of the ideal distribution ( $y$-axis), the ideal ratio is $10 \%$. Color bar shows Lipizzaner variant.

promoting diversity, consistently has the lowest absolute difference, i.e., of all methods, it best replicates the $10 \%$ frequency of images per class in the original distribution.

Finally, we investigate the diversity of the evolved networks to see whether one network is replicated over the entire grid and neighborhoods. We hypothesize that the max neighbor distance, $\Delta_{n}^{\max }$, property impacts the information exchange between the neighborhood sub-populations and 


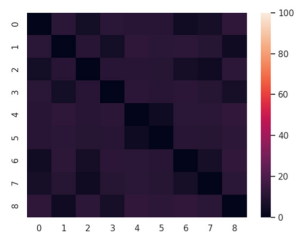

(a) $3 \times 3$ RAND-COMBOS

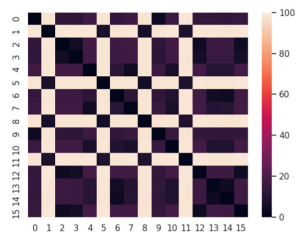

(e) $4 \times 4$ RAND-COMBOS

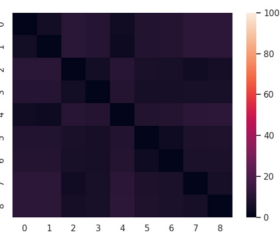

(b) $3 \times 3$ MSE-MSE

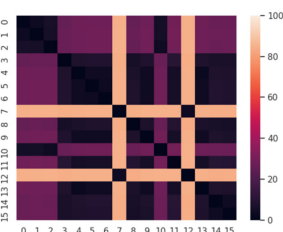

(f) $4 \times 4$ MSE-MSE

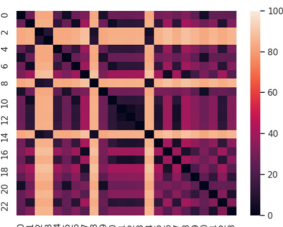

(i) $5 \times 5$ RAND-COMBOS

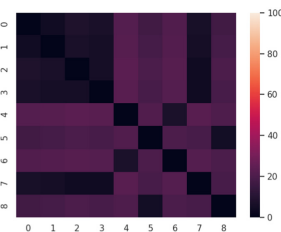

(c) $3 \times 3 \mathrm{BCE}-\mathrm{BCE}$

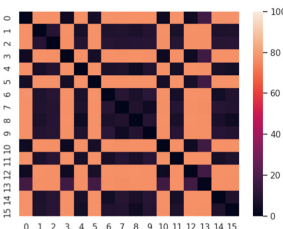

(g) $4 \times 4$ BCE-BCE

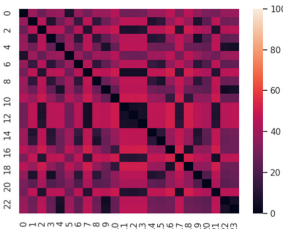

(j) $5 \times 5$ BCE-BCE

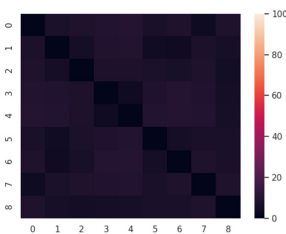

(d) $3 \times 3 \mathrm{HEU}-\mathrm{HEU}$

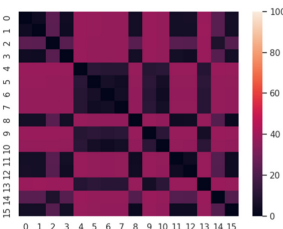

(h) $4 \times 4$ HEU-HEU

Fig. 9. Diversity of of population in genome space. Heatmap of $L_{2}$ distance between the generators in the population on MNIST at the final generation. X-axis and Y-axis show each grid cell, and dark indicates small distance.

Table 11. $L_{2}$ Distances on MNIST Dataset

\begin{tabular}{ccccc}
\hline Experiment & RAND-COMBOS & BCE-BCE & HEU-HEU & MSE-MSE \\
\hline$M N I S T-3 \times 3$ & $\mathbf{1 4 . 0 0 3}(6.659)$ & $13.850(8.291)$ & $6.426(2.739)$ & $7.320(3.565)$ \\
$M N I S T-4 \times 4$ & $\mathbf{4 3 . 6 8 1}(42.579)$ & $40.101(34.917)$ & $23.260(15.083)$ & $29.884(30.588)$ \\
$M N I S T-5 \times 5$ & $\mathbf{4 0 . 2 2 7}(29.700)$ & $31.984(15.313)$ & NA & NA
\end{tabular}

Mean (std) distance values between the weights of the networks in different cells, high values indicate more networks diversity in the grid. RAND-COMBOS consistently has the highest distance value. Highest value is in bold.

manifests itself in the diversity of the population's network parameters. The result for generators shows that there is no significant replication of networks across the generator population, as shown in Figure 9. We use the $L_{2}$ distance to measure the distance between neural network parameters; this is a rough measure that provides insight whether neural networks are identical or not. The diversity fluctuates across the setups and methods. In Table 11, we can observe that the average diversity increases when $\Delta_{n}^{\max }$ is greater than 0 . This is likely due to the increase in propagation time for the information exchange. In addition, RAND-COMBOS has the largest diversity variance for $4 \times 4$ and $5 \times 5$, i.e., there are some networks that are more diverse than others.

In summary, spatial grids with populations mapped across them exhibit diversity. This diversity can be increased by a supplementary method that randomizes loss functions used for training. The increase is due to the change in loss function impacting the training convergence of model parameters. 


\section{CONCLUSIONS \& FUTURE WORK}

In this article, we have introduced a scalable, robust, distributed GAN training framework called Lipizzaner. Lipizzaner combines the advantages of gradient-based optimization for GANs with those of coevolutionary systems and allows scaling over a distributed spatial grid topology. A relatively small spatial grid is sufficient to overcome the common limitations of GANs and improves performance compared to other evolutionary GAN training methods in the literature, due to the spatial separation and asynchronous evaluation. Moreover, the performance can improve with increased grid size. The utility of the Lipizzaner components was demonstrated by an ablation study.

In addition, we investigated diversity in the context of Lipizzaner's spatial coevolutionary (in particular, competitive) populations. We considered an explicit means to further promote diversity, which is to probabilistically choose one of three loss functions each round of training. This method, called RAND-COMBOS, was tested on the MNIST and CelebA datasets. It showed increased diversity across grid sizes in comparison to the other Lipizzaner methods. It also showed no appreciable loss in performance. Given it is often hard to know the loss function with which to train a GAN, RAND-COMBOS avoids an uninformed decision or exhaustive combinatoric experimentation.

The reported Lipizzaner CelebA FID scores are worse than from state-of-the-art GAN architectures [Lin et al. 2019; Unterthiner et al. 2017; Zhang et al. 2019b]. However, Lipizzaner can support any type of GAN, so we plan to experiment with more heterogeneous cell configurations, i.e., architectures. Finally, other advancements in evolutionary algorithms that can improve the robustness of GAN training, e.g., scheduled mutation rate changes over time or in response to population convergence, will be considered.

\section{REFERENCES}

Abdullah Al-Dujaili, Tom Schmiedlechner, Erik Hemberg, and Una-May O'Reilly. 2018a. Towards distributed coevolutionary GANs. In Proceedings of the AAAI Fall Symposium. arXiv preprint arXiv:1807.08194 (2018).

Abdullah Al-Dujaili, Shashank Srikant, Erik Hemberg, and Una-May O'Reilly. 2018b. On the application of Danskin's theorem to derivative-free minimax optimization. In Proceedings of the International Workshop on Global Optimization.

Martin Arjovsky and Léon Bottou. 2017. Towards principled methods for training generative adversarial networks. arXiv preprint arXiv:1701.04862 (2017).

Martin Arjovsky, Soumith Chintala, and Léon Bottou. 2017. Wasserstein generative adversarial networks. In Proceedings of the 34th International Conference on Machine Learning (ICML'17). JMLR.org, 214-223.

Sanjeev Arora, Rong Ge, Yingyu Liang, Tengyu Ma, and Yi Zhang. 2017. Generalization and equilibrium in generative adversarial nets (GANs). arXiv preprint arXiv:1703.00573 (2017).

Sanjeev Arora, Andrej Risteski, and Yi Zhang. 2018. Do GANs learn the distribution? Some theory and empirics. In Proceedings of the International Conference on Learning Representations. Retrieved from https://openreview.net/forum?id= BJehNfW0-.

Helio J. C. Barbosa. 1999. A coevolutionary genetic algorithm for constrained optimization. In Proceedings of the Congress on Evolutionary Computation. IEEE, 1605-1611.

Helio J. C. Barbosa. 1997. A coevolutionary genetic algorithm for a game approach to structural optimization. In Proceedings of the International Conference on Genetic Algorithms.

Ali Borji. 2019. Pros and cons of GAN evaluation measures. Comput. Vis. Image Underst. 179 (2019), 41-65.

Jürgen Branke and Johanna Rosenbusch. 2008. New approaches to coevolutionary worst-case optimization. In Proceedings of the International Conference on Parallel Problem Solving from Nature. Springer, 144-153.

Jason Brownlee. 2019. Generative Adversarial Networks with Python Deep Learning Generative Models for Image Synthesis and Image Translation. Machine Learning Mastery.

Andrés Camero, Jamal Toutouh, and Enrique Alba. 2020. Random error sampling-based recurrent neural network architecture optimization. Eng. Applic. Artif. Intell. 96 (2020), 103946.

Tatjana Chavdarova and François Fleuret. 2018. SGAN: An alternative training of generative adversarial networks. In Proceedings of the IEEE Conference on Computer Vision and Pattern Recognition. 9407-9415.

Keyang Cheng, Rabia Tahir, Lubamba Kasangu Eric, and Maozhen Li. 2020. An analysis of generative adversarial networks and variants for image synthesis on MNIST dataset. Multimedia Tools Applic. 79, 19 (2020), 13725-13752. 
Soumith Chintala, Emily Denton, Martin Arjovsky, and Michael Mathieu. 2016. How to train a GAN? Tips and tricks to make GANs work. Retrieved from https://github.com/soumith/ganhacks.

Dave Cliff and Geoffrey F. Miller. 1995. Tracking the red queen: Measurements of adaptive progress in co-evolutionary simulations. In Proceedings of the European Conference on Artificial Life. Springer, 200-218.

Victor Costa, Nuno Lourenço, and Penousal Machado. 2019. Coevolution of generative adversarial networks. In Proceedings of the International Conference on the Applications of Evolutionary Computation. Springer, 473-487.

Dario Floreano and Claudio Mattiussi. 2008. Bio-inspired Artificial Intelligence: Theories, Methods, and Technologies. The MIT Press.

Chi-Keong Goh and Kay Chen Tan. 2009. A Competitive-cooperative coevolutionary paradigm for dynamic multiobjective optimization. IEEE Trans. Evolut. Comput. 13, 1 (Feb. 2009), 103-127. DOI : https://doi.org/10.1109/TEVC.2008.920671

Ian Goodfellow. 2016. NIPS 2016 tutorial: Generative adversarial networks. arXiv preprint arXiv:1701.00160 (2016).

Ian Goodfellow, Jean Pouget-Abadie, Mehdi Mirza, Bing Xu, David Warde-Farley, Sherjil Ozair, Aaron Courville, and Yoshua Bengio. 2014. Generative adversarial nets. In Proceedings of the International Conference on Advances in Neural Information Processing Systems. 2672-2680.

Robin Harper. 2014. Evolving robocode tanks for Evo robocode. Gen. Prog. Evolv. Mach. 15, 4 (2014), 403-431.

Jeffrey W. Herrmann. 1999. A genetic algorithm for minimax optimization problems. In Proceedings of the Congress on Evolutionary Computation, Vol. 2. IEEE, 1099-1103.

Martin Heusel, Hubert Ramsauer, Thomas Unterthiner, and Bernhard Nessler. 2017a. GANs trained by a two time-scale update rule converge to a local Nash equilibrium. arXiv preprint arXiv:1706.08500 (2017).

Martin Heusel, Hubert Ramsauer, Thomas Unterthiner, Bernhard Nessler, Günter Klambauer, and Sepp Hochreiter. 2017b. GANs trained by a two time-scale update rule converge to a Nash equilibrium. arXiv preprint arXiv:1706.08500 (2017).

W. Daniel Hillis. 1990. Co-evolving parasites improve simulated evolution as an optimization procedure. Physica D: Nonlin Phenom. 42, 1 (1990), 228-234. DOI : https://doi.org/10.1016/0167-2789(90)90076-2

Babak Hodjat, Erik Hemberg, Hormoz Shahrzad, and Una-May O’Reilly. 2014. Maintenance of a long running distributed genetic programming system for solving problems requiring big data. In Genetic Programming Theory and Practice XI. Springer, 65-83.

Phil Husbands. 1994. Distributed coevolutionary genetic algorithms for multi-criteria and multi-constraint optimisation. In Proceedings of the AISB Workshop on Evolutionary Computing. Springer, 150-165.

Max Jaderberg, Valentin Dalibard, Simon Osindero, Wojciech M. Czarnecki, Jeff Donahue, Ali Razavi, Oriol Vinyals, Tim Green, Iain Dunning, Karen Simonyan, et al. 2017. Population based training of neural networks. arXiv preprint arXiv:1711.09846 (2017).

Mikkel T. Jensen. 2003. A new look at solving minimax problems with coevolutionary genetic algorithms. In Metaheuristics: Computer Decision-making. Springer, 369-384.

Daniel Jiwoong Im, He Ma, Chris Dongjoo Kim, and Graham Taylor. 2016. Generative adversarial parallelization. arXiv preprint arXiv:1612.04021 (2016).

Rodney W. Johnson, Michael E. Melich, Zbigniew Michalewicz, and Martin Schmidt. 2005. Coevolutionary optimization of fuzzy logic intelligence for strategic decision support. IEEE Trans. Evolut. Comput. 9, 6 (Dec. 2005), 682-694. DOI : https: //doi.org/10.1109/TEVC.2005.856208

Daniel Le Ly and Hod Lipson. 2014. Optimal experiment design for coevolutionary active learning. IEEE Trans. Evolut. Comput. 18, 3 (June 2014), 394-404. DOI : https://doi.org/10.1109/TEVC.2013.2281529

Yann LeCun. 1998. The MNIST database of handwritten digits. Retrieved from http://yann.lecun.com/exdb/mnist/.

Timothée Lesort, Andrei Stoian, Jean-François Goudou, and David Filliat. 2019. Training discriminative models to evaluate generative ones. In Proceedings of the International Conference on Artificial Neural Networks. Springer, 604-619.

Chengtao Li, David Alvarez-Melis, Keyulu Xu, Stefanie Jegelka, and Suvrit Sra. 2017a. Distributional adversarial networks. arXiv preprint arXiv:1706.09549 (2017).

Jerry Li, Aleksander Madry, John Peebles, and Ludwig Schmidt. 2017b. Towards understanding the dynamics of generative adversarial networks. arXiv preprint arXiv:1706.09884 (2017).

Chieh Hubert Lin, Chia-Che Chang, Yu-Sheng Chen, Da-Cheng Juan, Wei Wei, and Hwann-Tzong Chen. 2019. COCO-GAN: Generation by parts via conditional coordinating. In Proceedings of the IEEE International Conference on Computer Vision. 4512-4521.

Ziwei Liu, Ping Luo, Xiaogang Wang, and Xiaoou Tang. 2015. Deep learning face attributes in the wild. In Proceedings of the International Conference on Computer Vision (ICCV'15).

Ilya Loshchilov. 2013. Surrogate-assisted evolutionary algorithms. Ph.D. Dissertation. University Paris South Paris XI; National Institute for Research in Computer Science and Automatic-INRIA.

Mario Lucic, Karol Kurach, Marcin Michalski, Sylvain Gelly, and Olivier Bousquet. 2018. Are GANs created equal? A largescale study. In Proceedings of the International Conference on Advances in Neural Information Processing Systems. 700-709. 
Xudong Mao, Qing Li, Haoran Xie, Raymond Y. K. Lau, Zhen Wang, and Stephen Paul Smolley. 2017. Least squares generative adversarial networks. In Proceedings of the IEEE International Conference on Computer Vision. 2794-2802.

L. Miguel Antonio and C. A. Coello Coello. 2018. Coevolutionary multiobjective evolutionary algorithms: Survey of the state-of-the-art. IEEE Trans. Evolut. Comput. 22, 6 (Dec. 2018), 851-865. DOI : https://doi.org/10.1109/TEVC.2017.2767023

Melanie Mitchell. 2006. Coevolutionary learning with spatially distributed populations. Comput. Intell.: Princ. Pract. 400, 7 (2006), 137-152.

Nick Moran and Jordan Pollack. 2018. Coevolutionary neural population models. In Artificial Life Conference Proceedings. The MIT Press, 39-46.

Gonçalo Mordido, Haojin Yang, and Christoph Meinel. 2018. Dropout-GAN: Learning from a dynamic ensemble of discriminators. arXiv preprint arXiv:1807.11346 (2018).

Behnam Neyshabur, Srinadh Bhojanapalli, and Ayan Chakrabarti. 2017. Stabilizing GAN training with multiple random projections. arXiv preprint arXiv:1705.07831 (2017).

Tu Nguyen, Trung Le, Hung Vu, and Dinh Phung. 2017. Dual discriminator generative adversarial nets. In Proceedings of the International Conference on Advances in Neural Information Processing Systems. 2670-2680.

Stefano Nolfi and Dario Floreano. 1998. Coevolving predator and prey robots: Do "arms races" arise in artificial evolution? Artif. Life 4, 4 (1998), 311-335.

Elena Popovici, Anthony Bucci, R. Paul Wiegand, and Edwin D. De Jong. 2012. Coevolutionary principles. In Handbook of Natural Computing. Springer, 987-1033.

Xin Qiu, Jian-Xin Xu, Yinghao Xu, and Kay Chen Tan. 2017. A new differential evolution algorithm for minimax optimization in robust design. IEEE Trans. Cyber. 48, 5 (2017), 1355-1368.

Alec Radford, Luke Metz, and Soumith Chintala. 2015. Unsupervised representation learning with deep convolutional generative adversarial networks. arXiv preprint arXiv:1511.06434 (2015).

Christopher D. Rosin and Richard K. Belew. 1997. New methods for competitive coevolution. Evolut. Comput. 5, 1 (1997), 1-29.

Tim Salimans, Jonathan Ho, Xi Chen, and Ilya Sutskever. 2017. Evolution strategies as a scalable alternative to reinforcement learning. arXiv:1703.03864 (2017).

Tom Schmiedlechner. 2018. Industrial-scale Evolutionary Machine Learning. Master's thesis. University of Applied Sciences Upper Austria.

Tom Schmiedlechner, Ignavier Ng Zhi Yong, Abdullah Al-Dujaili, Erik Hemberg, and Una-May O’Reilly. 2018. Lipizzaner: A system that scales robust generative adversarial network training, In Proceedings of the 32nd Conference on Neural Information Processing Systems (NeurIPS'18) Workshop on Systems for ML and Open Source Software. arXiv preprint arXiv:1811.12843 (2018).

Konstantin Shmelkov, Cordelia Schmid, and Karteek Alahari. 2018. How good is my GAN? In Proceedings of the European Conference on Computer Vision (ECCV'18). 213-229.

Kenneth O. Stanley and Jeff Clune. 2017. Welcoming the Era of Deep Neuroevolution-Uber Engineering Blog. Retrieved from https://eng.uber.com/deep-neuroevolution/.

Kenneth O. Stanley, Jeff Clune, Joel Lehman, and Risto Miikkulainen. 2019. Designing neural networks through neuroevolution. Nat. Mach. Intell. 1, 1 (2019), 24-35.

Kenneth O. Stanley and Risto Miikkulainen. 2002. Evolving neural networks through augmenting topologies. Evolut. Comput. 10, 2 (2002), 99-127.

Guy Tevet, Gavriel Habib, Vered Shwartz, and Jonathan Berant. 2018. Evaluating text GANs as language models. arXiv preprint arXiv:1810.12686 (2018).

Ilya O. Tolstikhin, Sylvain Gelly, Olivier Bousquet, Carl-Johann Simon-Gabriel, and Bernhard Schölkopf. 2017. Adagan: Boosting generative models. In Proceedings of the International Conference on Advances in Neural Information Processing Systems. 5430-5439.

Jamal Toutouh, Erik Hemberg, and Una-May O’Reilly. 2019. Spatial evolutionary generative adversarial networks. In Proceedings of the Genetic and Evolutionary Computation Conference (GECCO'19). Association for Computing Machinery, New York, NY, 472-480. DOI : https://doi.org/10.1145/3321707.3321860

Jamal Toutouh, Erik Hemberg, and Una-May O'Reilly. 2020. Data dieting in GAN training. In Deep Neural Evolution. Springer, 379-400.

Thomas Unterthiner, Bernhard Nessler, Calvin Seward, Günter Klambauer, Martin Heusel, Hubert Ramsauer, and Sepp Hochreiter. 2017. Coulomb GANs: Provably optimal Nash equilibria via potential fields. arXiv preprint arXiv:1708.08819 (2017).

Chaoyue Wang, Chang Xu, Xin Yao, and Dacheng Tao. 2019. Evolutionary generative adversarial networks. IEEE Trans. Evolut. Comput. 23, 6 (2019), 921-934.

Yaxing Wang, Lichao Zhang, and Joost van de Weijer. 2016. Ensembles of generative adversarial networks. arXiv preprint arXiv:1612.00991 (2016). 
Richard A. Watson and Jordan B. Pollack. 2001. Coevolutionary dynamics in a minimal substrate. In Proceedings of the 3rd Conference on Genetic and Evolutionary Computation. Morgan Kaufmann Publishers Inc., 702-709.

Daan Wierstra, Tom Schaul, Jan Peters, and Juergen Schmidhuber. 2008. Natural evolution strategies. In Proceedings of the IEEE Congress on Evolutionary Computation (CEC'08). IEEE, 3381-3387.

Nathan Williams and Melanie Mitchell. 2005. Investigating the success of spatial coevolution. In Proceedings of the 7th Conference on Genetic and Evolutionary Computation. ACM, 523-530.

Steven R. Young, Derek C. Rose, Travis Johnston, William T. Heller, Thomas P. Karnowski, Thomas E. Potok, Robert M. Patton, Gabriel Perdue, and Jonathan Miller. 2017. Evolving deep networks using HPC. In Proceedings of the Machine Learning on HPC Environments Conference. ACM, 7.

Han Zhang, Zizhao Zhang, Augustus Odena, and Honglak Lee. 2019b. Consistency regularization for generative adversarial networks. arXiv preprint arXiv:1910.12027 (2019).

Xin-Yuan Zhang, Yue-Jiao Gong, Ying Lin, Jie Zhang, Sam Kwong, and Jun Zhang. 2019a. Dynamic cooperative coevolution for large scale optimization. IEEE Trans. Evolut. Comput. (2019), 1-1. DOI : https://doi.org/10.1109/TEVC.2019.2895860

Junbo Zhao, Michael Mathieu, and Yann LeCun. 2016. Energy-based generative adversarial network. arXiv preprint arXiv:1609.03126 (2016).

Received October 2019; revised March 2021; accepted March 2021 